SFB/CPP-09-86

TTP09-33

\title{
Gluinonia: Energy Levels, Production and Decay
}

\author{
Matthias R. Kauth, Johann H. Kühn, Peter Marquard and Matthias Steinhauser \\ Institut für Theoretische Teilchenphysik \\ Karlsruhe Institute of Technology (KIT) \\ 76128 Karlsruhe, Germany
}

\begin{abstract}
Predictions for energy levels, production and decay rate of gluinonia, nonrelativistic boundstates of gluinos, are presented. The potential between color-octet constituents is derived in next-to-next-to leading order and one-loop QCD corrections are derived for the production cross section and the decay rate into gluon jets. In addition we evaluate the decay rate into top quarks and into two photons. The signal-to-background ratio is estimated for the dominant decay mode and found to be around $0.5 \%$. For relatively light gluinos the bound states thus might be detectable.
\end{abstract}

PACS numbers: 12.38.Bx 12.60.Jv 14.80.Ly 


\section{Introduction}

With the turning-on of the LHC in the near future, one of the most important tasks of both general-purpose detectors ATLAS and CMS will be the search for physics beyond the Standard Model, with one of the most promising candidates being Supersymmetry. In the course of this program, which will obviously be adjusted and focused with increasing luminosity and with the first indications of "New Physics", the determination of the properties of newly detected particles will be a major task. Indeed, only after the quantum numbers, the interaction and the mass of a new particle have been measured with high precision, this requirement can be considered fulfilled.

In the present paper we want to concentrate on the gluino, the supersymmetric partner of the gluon, and discuss the possibility to determine its quantum numbers, i.e. its spin, color, Majorana nature and its mass, through the investigation of non-relativistic boundstates of two gluinos, generally denoted "gluinonia". The classification of gluinonia according to their quantum numbers and their qualitative properties, i.e. spectra and decay modes, as well as estimates of their production cross section at hadron colliders have been studied already more than two decades ago and presented in Refs. [1-3] concentrating at that time on gluino masses which are by now experimentally excluded. More recently gluinonia have been discussed in Refs. $[4,5]$. Hadronic transitions between different gluinonium levels and some rare annihilation decays were evaluated in [4]. The production of vector and pseudoscalar states at the Tevatron and the LHC was investigated in [5] where it was argued that, given sufficiently good yet realistic jet mass resolution, pseudoscalar states could be detected for bound state masses up to $3 \mathrm{TeV}$. A phenomenological study of the question, to which extent a signal for color-octet states could indeed be detected experimentally, has been performed in [6]. In all these cases, however, the production cross section has been calculated in lowest order approximation only and a qualitative phenomenological potential for the gluino-gluino interaction has been employed. The topic has gained renewed interest in connection with Split SUSY [7,8], (see, e.g., Refs. [9, 10]).

The detection of these bound states would provide important information about gluino properties, difficult to obtain through other means. Since gluinonia only exist if gluinos do not decay too rapidly, say with decay rates less than a few $\mathrm{GeV}$, their observation would immediately provide at least an upper limit on $\Gamma_{\tilde{g}}$. The energy spectrum of the gluinonium system, e.g. the mass difference between ground state and first radial excitation (or open gluino production) would be sensitive to the potential and thus to the color-octet nature of the constituents. The same is true for the production cross section which is proportional to the square of the wave function at the origin. Gluinos are expected to decay through cascades into several jets plus the lightest supersymmetric particle (LSP) which escapes detection. For this reason the precise determination of its mass in direct gluino decays is limited by the missing mass or energy resolution.

In contrast, once the mass of the boundstate and the dynamics of the interaction are known with sufficient precision, the mass of the constituents is fixed with the corresponding 
accuracy and in any case subject to a different systematic error.

The energy levels of gluinonia were estimated already in the early papers employing phenomenological color-octet potentials, obtained from phenomenological quarkonium potentials by multiplication with the ratio of the corresponding Casimir operators $C_{A} / C_{F}=9 / 4$ and the gluino mass was interpreted as an effective mass closely related to the pole mass. During recent years more refined studies have been performed to describe non-relativistic boundstates of color-triplet constituents. This includes the evaluation of the static twoloop potential [11-13], first steps toward the evaluation of the three-loop potential [14] and the calculation of mass-suppressed terms proportional $\alpha_{s}^{2} / m$ [15-19] and $\alpha_{s} / m^{2}[17,19-21]$. Mass definitions better suited to control the long distance part of the potential have been introduced $[22,23]$, and the evaluation of the spectra through analytic methods has been possible, using a perturbative approach to include higher order contributions from the potential [24-26]. Last but not least, finite-width effects were incorporated for top-quark production at electron-positron [27-33] and hadron colliders [34-36]. These new results can be applied also in studies of gluino bound states.

In order to arrive at reliable predictions for the signals of gluinonium production and decay, the inclusion of a realistic QCD potential and next-to-leading order (NLO) corrections to boundstate production at the LHC is mandatory. Considering and building on the significant progress in the perturbative treatment of non-relativistic boundstates, in particular quarkonium and positronium, predictions of similar quality can be made for boundstates with color-octet constituents. This is the main content of the present paper.

We start with a brief overview and a discussion of the quantum numbers and qualitative properties of boundstates with Majorana constituents and evaluate the QCD potential for a color-octet boundstate (Section 21). Subsequently, in Section 3, we calculate the spectrum and the wave function at the origin for the lowest-lying levels.

These quantities are the necessary ingredients for a realistic prediction of gluinonium production and decay rates presented in Section 4. Assuming that squarks are relatively heavy, the dominant decay channel of the pseudoscalar singlet boundstate proceeds through two gluons, and it will be a difficult task to distinguish this signal from the irreducible background from the two-gluon jet continuum.

In Section 5 we consider as an alternative the decays into top quarks and two photons, which can proceed through virtual quark $(q)$ and squark $(\tilde{q})$ intermediate states. One might, in principle, hope that the extremely clean $\gamma \gamma$ signal sticks out of the irreducible background similarly to the Higgs decay into two photons which has been identified as a promising signal significantly superior to the $b \bar{b}$ or two-gluon mode. Similar considerations apply to the $t \bar{t}$ mode.

In order that these modes can compete with the two-gluon channel, squarks should not be dramatically heavier than gluinos. On the other hand, to avoid dominance of the singlegluino decay, the decay mode $\tilde{g} \rightarrow \tilde{q} \bar{q}$ should be kinematically forbidden, corresponding to $m_{\tilde{g}} \leq m_{\tilde{q}}$. We therefore evaluate the corresponding decay rates and study if, given suitable 


\begin{tabular}{c|ccc}
$\mathrm{R}$ & $\left(F^{R}\right)^{2}$ & $F^{a, 1} \cdot F^{a, 2}$ & interaction \\
\hline $1_{s}$ & 0 & -3 & attractive \\
$8_{s}, 8_{a}$ & 3 & $-\frac{3}{2}$ & attractive \\
$10_{a}, \overline{10}_{a}$ & 6 & 0 & neutral \\
$27_{s}$ & 8 & 1 & repulsive
\end{tabular}

Table 1: Color interaction of two SU(3) octets.

choices for the respective masses, the two-photon mode might lead to a possible signal. In Section [6 a rough estimate of the dominant signal versus background is presented. Section 7 contains our conclusions.

\section{Properties of gluinonia}

Let us start with a brief recapitulation of the quantum numbers of gluino boundstates and the corresponding color, spin and orbital momentum configurations [1-3]. These differ from those of quarkonia due to the restrictions arising from the Majorana nature of gluinos, and due to their different color assignment.

Two color-octet states can be combined into irreducible representations as follows

$$
8 \otimes 8=1_{s} \oplus 8_{s} \oplus 8_{a} \oplus 10_{a} \oplus \overline{10}_{a} \oplus 27_{s},
$$

where the index indicates the (anti-)symmetry with respect to their color index. The interaction can be either attractive, repulsive or absent (in lowest order). In lowest order the coefficient of the QCD potential is given by the expectation value of the product of the color generators $F_{i j}^{a} F_{k l}^{a}$, taken between two-particle states in the respective representation.

This product, in turn, can be expressed by the eigenvalues of the quadratic Casimir operator of the constituents, $C_{A}=3$, and the boundstate in representation $R, C_{R}=$ $\left(F^{R}\right)^{2}$ :

$$
F^{a, 1} \cdot F^{a, 2}=\frac{1}{2}\left[\left(F^{R}\right)^{2}-\left(F^{a, 1}\right)^{2}-\left(F^{a, 2}\right)^{2}\right]=\frac{1}{2}\left(C_{R}-2 C_{A}\right) .
$$

The results are listed in Tab. 1, In the following we shall limit the discussion to the cases with negative coefficients, corresponding to attraction.

Fermi statistics and the Majorana nature of the gluinos lead to additional restrictions. For the symmetric color configurations $1_{s}$ and $8_{s}$ antisymmetric spin-angular momentum wave functions, $(-1)^{L+S}=1$, are required, for the antisymmetric color configuration $8_{a}$ symmetric ones, $(-1)^{L+S}=-1$. The intrinsic parity of a Majorana particle can be chosen to be imaginary, its parity under charge reflection real, leading to negative intrinsic parity

and positive charge parity of the boundstate. For a few lowest orbital angular momenta the complete set of boundstate quantum numbers is listed in Tab. 2. 


\begin{tabular}{c|ccccccc}
${ }^{2 S+1} L_{J}$ & ${ }^{1} S_{0}$ & ${ }^{3} S_{1}$ & ${ }^{1} P_{1}$ & ${ }^{3} P_{0}$ & ${ }^{3} P_{1}$ & ${ }^{3} P_{2}$ & ${ }^{1} D_{2}$ \\
\hline $\mathrm{L}$ & 0 & 0 & 1 & 1 & 1 & 1 & 2 \\
$\mathrm{~S}$ & 0 & 1 & 0 & 1 & 1 & 1 & 0 \\
$(\tilde{g} \tilde{g})_{s}$ & $0^{-+}$ & - & - & $0^{++}$ & $1^{++}$ & $2^{++}$ & $2^{-+}$ \\
$(\tilde{g} \tilde{g})_{a}$ & - & $1^{-+}$ & $1^{++}$ & - & - & - & -
\end{tabular}

Table 2: Lowest-lying states $J^{P C}$ of the gluinonium spectrum.

The production rate of non-relativistic boundstates in hard collisions is proportional to the squared wave function at the origin (for $S$ waves) or its derivative (for $P$ waves), the latter being significantly suppressed and of relative order $v^{2}$. For this reason the following discussion will be limited to $S$ waves only. We, furthermore, anticipate that the experimental mass resolution and, eventually, the large natural width of the boundstate will lead to a sizable smearing of the "narrow" resonances. The signal to background ratio will be small and their detection difficult. For this reason we will consider color-singlet states only, where the level spacings and production rates are enhanced approximately by the square of the color coefficient listed in Tab. 1.

Let us now discuss the QCD potential. Similarly to the case of heavy-quark boundstates it can be decomposed into the following two terms

$$
V_{\tilde{\mathrm{g}} \tilde{\mathrm{g}}}(\vec{r})=V_{\mathrm{C}}(r)+V_{\mathrm{nC}}(\vec{r})
$$

which are ordered according to their inverse powers in the constituent mass $m$ and are given in coordinate space in close similarity to the quarkonium potential (see e.g. [19]). For $S$ waves it reads

$$
\begin{aligned}
V_{\mathrm{C}}(r)= & -\frac{C_{A} \alpha_{s}\left(\mu^{2}\right)}{r}\left\{1+\frac{\alpha_{s}\left(\mu^{2}\right)}{4 \pi}\left[a_{1}+2 \gamma_{E} \beta_{0}+2 \beta_{0} \ln (\mu r)\right]\right. \\
& +\frac{\alpha_{s}\left(\mu^{2}\right)^{2}}{(4 \pi)^{2}}\left[a_{2}+2 \gamma_{E}\left(2 a_{1} \beta_{0}+\beta_{1}\right)+\beta_{0}^{2}\left(\frac{\pi^{2}}{3}+4 \gamma_{E}^{2}\right)\right. \\
& \left.\left.+\left(4 a_{1} \beta_{0}+2 \beta_{1}+8 \gamma_{E} \beta_{0}^{2}\right) \ln (\mu r)+4 \beta_{0}^{2} \ln (\mu r)^{2}\right]\right\}, \\
V_{\mathrm{nC}}(\vec{r})= & U_{1}(r)+U_{2}(\vec{r})+U_{3}(r), \\
U_{1}(r)= & -\frac{\Delta^{2}}{4 m_{\tilde{g}}^{3}}+\frac{C_{A} \alpha_{s}\left(\mu^{2}\right)}{m_{\tilde{g}}^{2} r} \Delta, \\
U_{2}(\vec{r})= & \frac{4 \pi C_{A} \alpha_{s}\left(\mu^{2}\right)}{3 m_{\tilde{g}}^{2}} \vec{S}^{2} \delta(\vec{r}) \\
U_{3}(r)= & -\frac{C_{A}^{2} \alpha_{s}^{2}\left(\mu^{2}\right)}{4 m_{\tilde{g}} r^{2}}
\end{aligned}
$$


where

$$
\begin{aligned}
\beta_{0}= & \frac{11}{3} C_{A}-\frac{4}{3} T_{F} n_{f}, \\
\beta_{1}= & \frac{34}{3} C_{A}^{2}-\frac{20}{3} C_{A} T_{F} n_{f}-4 C_{F} T_{F} n_{f}, \\
a_{1}= & \frac{31}{9} C_{A}-\frac{20}{9} T_{F} n_{f}, \\
a_{2}= & {\left[\frac{4343}{162}+4 \pi^{2}-\frac{\pi^{4}}{4}+\frac{22}{3} \zeta(3)\right] C_{A}^{2}-\left[\frac{1798}{81}+\frac{56}{3} \zeta(3)\right] C_{A} T_{F} n_{f} } \\
& -\left[\frac{55}{3}-16 \zeta(3)\right] C_{F} T_{F} n_{f}+\left(\frac{20}{9} T_{F} n_{f}\right)^{2} .
\end{aligned}
$$

Here $\alpha_{s}$ stands for the strong coupling constant in the $\overline{\mathrm{MS}}$ scheme, $n_{f}$ is the number of active quark flavors, $C_{A}=3, C_{F}=4 / 3, T_{F}=1 / 2, \gamma_{E}=0.5772 \ldots$ the Euler constant, $\zeta(3)=1.2021 \ldots$ and $m_{\tilde{g}}$ is the constituent pole mass.

For the Coulomb part $V_{\mathrm{C}}$ the transformation from color-triplet, where the result can be found in the literature, to constituents in an arbitrary representation $R$, bound to a singlet state, can be understood as follows: In LO the overall normalization is changed by substituting $C_{F} \rightarrow C_{R}$. This is the only modification also in NLO, thus leaving the constant $a_{1}$ and the terms proportional to $\beta_{0}$ unchanged. For the case under discussion $(R=A)$ this is evident from Fig. 1: Diagram (a) contributes proportional $C_{R}^{2}$, diagram (b) proportional $C_{R}^{2}-\frac{1}{2} C_{R} C_{A}$. Its $C_{R}^{2}$ part can be combined with (a) and in total the ladder plus crossed ladder can be collected in the $C_{R} / r$ part of the potential. The $C_{R} C_{A}$ part of diagram (b) contributes to the $C_{A}$ term in $\beta_{0}$ and in the constant $a_{1}$. Diagram (d) contributes proportional $C_{R} T_{F} n_{f}$ and is responsible for the $T_{F} n_{f}$ terms in $\beta_{0}$ and $a_{1}$. The corresponding considerations are also applicable to the two-loop case, see e.g. Fig. 2 , For diagrams with virtual gluons only (e.g. Fig. 2 (a)), the substitution $C_{F} \rightarrow C_{R}$ is valid throughout. For diagrams with exactly one dressed gluon propagator exchange (e.g. Fig. 2 (c), (d), (e)), the same substitution is valid, as far as the coupling of the gluon to the constituent is concerned and only the external $C_{F}$ is replaced by $C_{R}$. Diagrams with three-gluon coupling through a triangular fermion loop (Fig. 2 (b)) are also proportional to $C_{R} C_{A} T_{F} n_{f}$ and it is again the first factor, which is chosen to be $C_{F}$ in the case of quarkand $C_{A}$ in the case of gluino-constituents. Diagrams similar to those of Fig. 1 (a)-(c), but with one dressed gluon (e.g. Fig. 2 (f)) can be handled like the NLO diagrams. In total, up to NNLO, the Coulomb part of the potential for the binding of constituents in an arbitrary representation $R$ is obtained through the substitution $C_{F} \rightarrow C_{R}$ in the overall factor 1

The term $U_{3}$ of Eq. (4) of order $\alpha_{s}^{2} / m$ originates from a one-loop calculation, and the factor $C_{F}\left(C_{F} / 2-C_{A}\right)$ must be replaced by $C_{R}\left(C_{R} / 2-C_{A}\right)$. The terms of order $\alpha_{s} / m^{2}$

\footnotetext{
${ }^{1}$ We do not expect that this statement holds true to arbitrary high orders. In contrast to heavy quarks gluinos together with gluons can form color-singlet states and even in quenched QCD the static potential between two gluinos is not expected to be confining.
} 


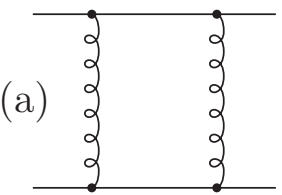

(b)

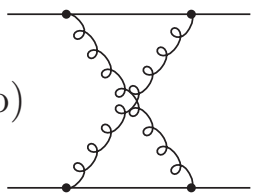

(c)

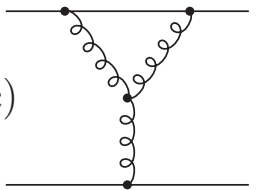

(d)

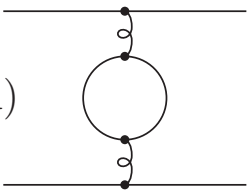

Figure 1: Typical NLO contributions to the $q \bar{q}$ potential. Straight and curely lines represent quarks and gluons, respectively.

(a)

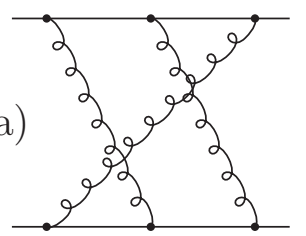

(d)

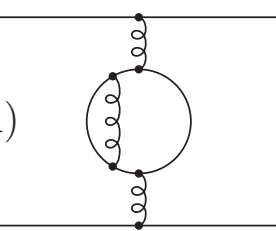

(b)

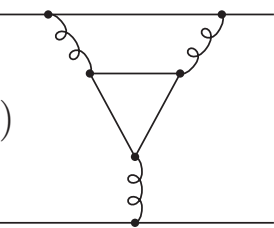

(e)

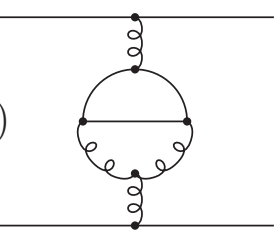

(c)

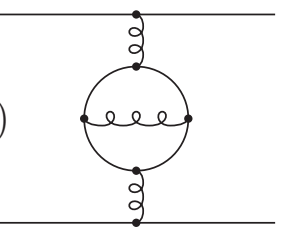

(f)

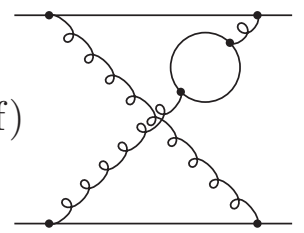

Figure 2: Typical NNLO contributions to the $q \bar{q}$ potential. The same notation as in Fig. 1 has been adopted.

collected in $U_{1}$ and $U_{2}$ can be translated simply by replacing the global factor $C_{F}$ by $C_{R}$. For our application the substitution $C_{R} \rightarrow C_{A}$ is implicit.

\section{Spectroscopy}

Using the potential as specified in Eqs. (3) and (44), binding energies, level spacings and bound-state wave functions are easily obtained by solving Schrödinger's equation numerically for $V_{C}$ and adding the singular terms collected in $U_{1}, U_{2}$ and $U_{3}$ in perturbation theory or, alternatively, by evaluating energy level and wave functions in a perturbative series in $\alpha_{s}$.

The analytic results both for binding energies $E_{n}$ and wave functions at the origin $\left|\Psi_{n}(0)\right|^{2}$ are listed in Appendix A. In numerical form the results for the two lowest levels are given 
by

$$
\begin{aligned}
E_{1}=-\frac{m_{\tilde{g}} C_{A}^{2} \alpha_{s}^{2}}{4}\{1 & +\alpha_{s}\left(2.44 L_{1}+3.20\right) \\
& \left.+\alpha_{s}^{2}\left[\left(4.47 L_{1}^{2}+9.71 L_{1}+12.47\right)_{C}+(20.81)_{n C}\right]\right\}, \\
E_{2}=-\frac{m_{\tilde{g}} C_{A}^{2} \alpha_{s}^{2}\{}{16}\left\{1+\alpha_{s}\left(2.44 L_{2}+4.42\right)\right. & \left.+\alpha_{s}^{2}\left[\left(4.47 L_{2}^{2}+14.19 L_{2}+20.54\right)_{C}+(11.95)_{n C}\right]\right\}, \\
\left|\Psi_{1}(0)\right|^{2}=\frac{m_{\tilde{g}}^{3} C_{A}^{3} \alpha_{s}^{3}\{1}{8 \pi}+\alpha_{s}\left(3.66 L_{1}-0.43\right) & \left.+\alpha_{s}^{2}\left[\left(8.93 L_{1}^{2}-5.11 L_{1}+5.83\right)_{C}+\left(27 L_{1}+57.38\right)_{n C}\right]\right\}, \\
\left|\Psi_{2}(0)\right|^{2}=\frac{m_{\tilde{g}}^{3} C_{A}^{3} \alpha_{s}^{3}\{1}{64 \pi}+\alpha_{s}\left(3.66 L_{2}-0.18\right) & \left.+\alpha_{s}^{2}\left[\left(8.93 L_{2}^{2}-3.86 L_{2}+10.19\right)_{C}+\left(27 L_{2}+29.53\right)_{n C}\right]\right\},
\end{aligned}
$$

with $L_{n}=\ln \left(n \mu /\left(m_{\tilde{g}} C_{A} \alpha_{s}\right)\right)$ and $m_{\tilde{g}}$ being the gluino pole mass. The first of the $\alpha_{s}^{2}$ terms give the Coulombic corrections $(\mathrm{C})$ the second ones the non-Coulombic ones $(\mathrm{nC})$. Here and below we use $\alpha_{s}=\alpha_{s}(\mu)$ as defined in the $\overline{\mathrm{MS}}$ scheme and the $\mu$ dependence from the numerical solution of the three loop renormalization group equation with the starting value $\alpha_{s}\left(M_{Z}\right)=0.1176$. We use $n_{f}=5$. This is well justified, as long as the characteristic scale $\alpha_{s} m_{\tilde{g}}$ is smaller than the top-quark mass, an assumption well justified for all gluino masses under consideration. Top-quark effects could be included following [37,38]. If not stated otherwise, in the following we use for the renormalization scale $\mu=\mu_{S}$ with $\mu_{S}=m_{\tilde{g}} C_{A} \alpha_{s}\left(\mu_{S}\right) / n$ corresponding to $L_{n}=0$.

We have convinced ourselves that the perturbative series and the numerical results are in very good agreement as far as $V_{\mathrm{C}}$ is concerned. The subsequent analysis will therefore be based on the fully perturbative approach.

The groundstate energy $E_{1}$ is shown in Fig. 3 as a function of $2 m_{\tilde{g}}$ for gluino masses $m_{\tilde{g}}$ between $300 \mathrm{GeV}$ and $1500 \mathrm{GeV}$. The LO (dotted), NLO (dashed) and NNLO predictions are displayed individually. For the NNLO result both the result for $V_{\mathrm{C}}$ (dash-dotted) and the one including the corrections collected in $U_{1}, U_{2}$ and $U_{3}$ (solid) are shown. As stated above, $m_{\tilde{g}}$ is understood as the constituent pole mass. The poor convergence of the perturbative series can partly be traced back to the large non-Coulombic correction which appear for the first time in NNLO and amount to twice the NNLO Coulombic one. Qualitatively this behavior is quite similar to the one observed for quarkonia in $[25,39,40]$. It remains to be seen to which extent inclusion of orders of $\mathrm{N}^{3} \mathrm{LO}$ and higher will stabilize these predictions 2 In Fig. 4 we show as an alternative representation the predictions for the ground state energy, using as reference the potential subtracted mass [22], which is

\footnotetext{
${ }^{2}$ For the quarkonium case, see Refs. [25, 41].
} 


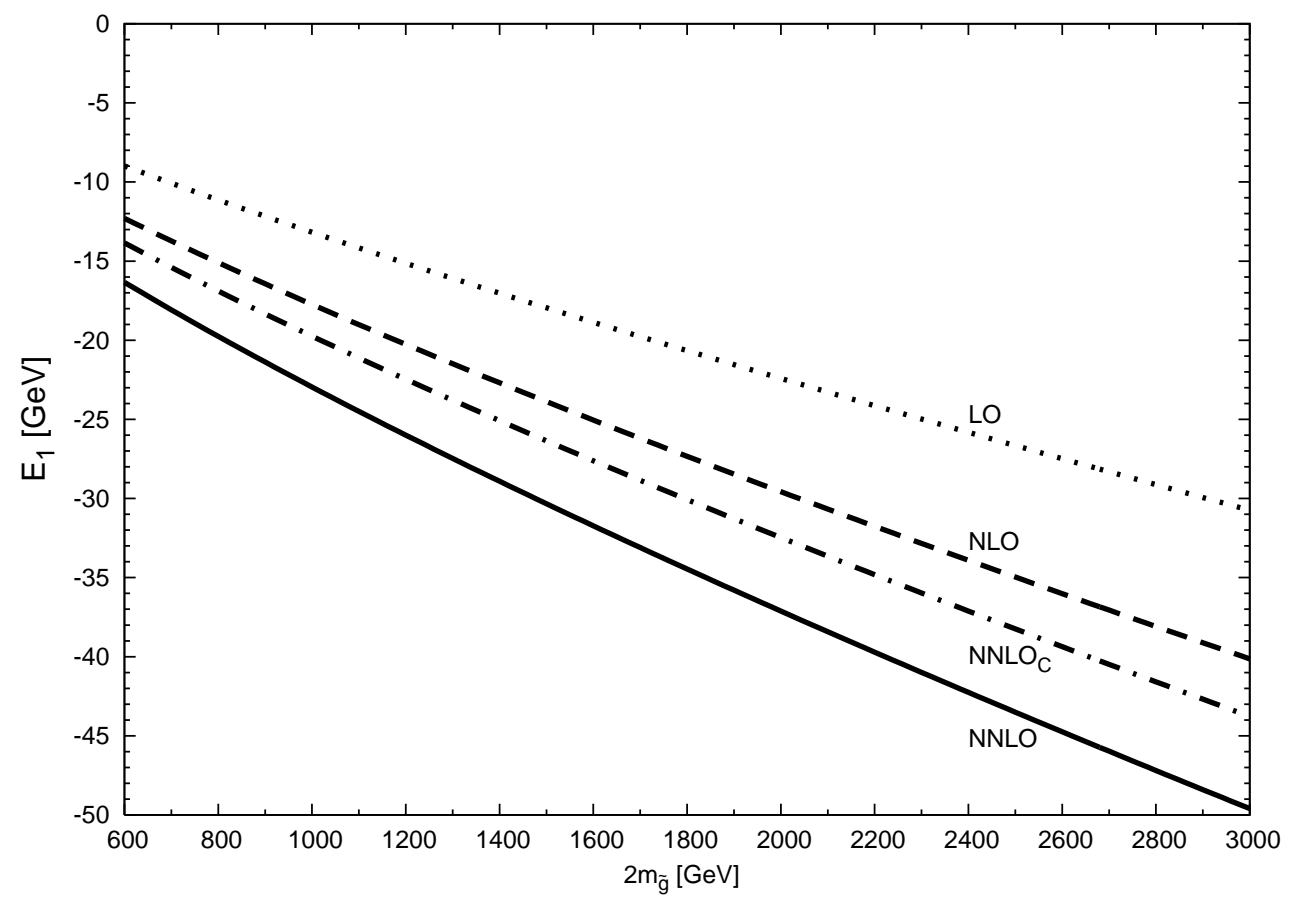

Figure 3: Ground state energy $E_{1}$ in the pole mass scheme as function of twice the constituent mass.

related to the pole mass through

$$
\begin{aligned}
m_{\tilde{g}}-m_{\mathrm{PS}}\left(\mu_{f}\right)= & \delta m\left(\mu_{f}\right)=-\frac{1}{2} \int_{|\vec{q}|<\mu_{f}} \frac{d^{3} q}{(2 \pi)^{3}} \tilde{V}(q) \\
= & \frac{C_{A} \alpha_{s}(\mu)}{\pi} \mu_{f}\left\{1+\frac{\alpha_{s}(\mu)}{4 \pi}\left[a_{1}-\beta_{0}\left(\ln \left(\frac{\mu_{f}^{2}}{\mu^{2}}\right)-2\right)\right]\right. \\
& +\left(\frac{\alpha_{s}(\mu)}{4 \pi}\right)^{2}\left[a_{2}-\left(2 a_{1} \beta_{0}+\beta_{1}\right)\left(\ln \left(\frac{\mu_{f}^{2}}{\mu^{2}}\right)-2\right)\right. \\
& \left.\left.+\beta_{0}^{2}\left(\ln ^{2}\left(\frac{\mu_{f}^{2}}{\mu^{2}}\right)-4 \ln \left(\frac{\mu_{f}^{2}}{\mu^{2}}\right)+8\right)\right]\right\} .
\end{aligned}
$$

In the following we adopt for the factorization scale $\mu_{f}=m_{\tilde{g}} C_{A} \alpha_{s}\left(\mu_{S}\right)$, independent of $n$ (with $\mu_{S}=m_{\tilde{g}} C_{A} \alpha_{s}\left(\mu_{S}\right) / n$ as before). (Note, that $m_{\mathrm{PS}}$ now also depends on $n$.) We refrain from listing explicitly the results for the energy levels in the potential subtracted scheme since the corresponding formulae are quite bulky. They can easily be obtained from Eqs. (6) and (17).

The implementation of the potential subtracted mass leads to a significantly improved convergence of the perturbative series for $E_{1}^{\mathrm{PS}}$ as far as the Coulombic part is concerned (see Fig. (4) and similarly for $E_{2}^{\mathrm{PS}}$. However, the meson mass difference between the $1 S$ and the $2 S$ state, $M(2 S)-M(1 S)$, is independent of the choice of the scheme and exhibits 


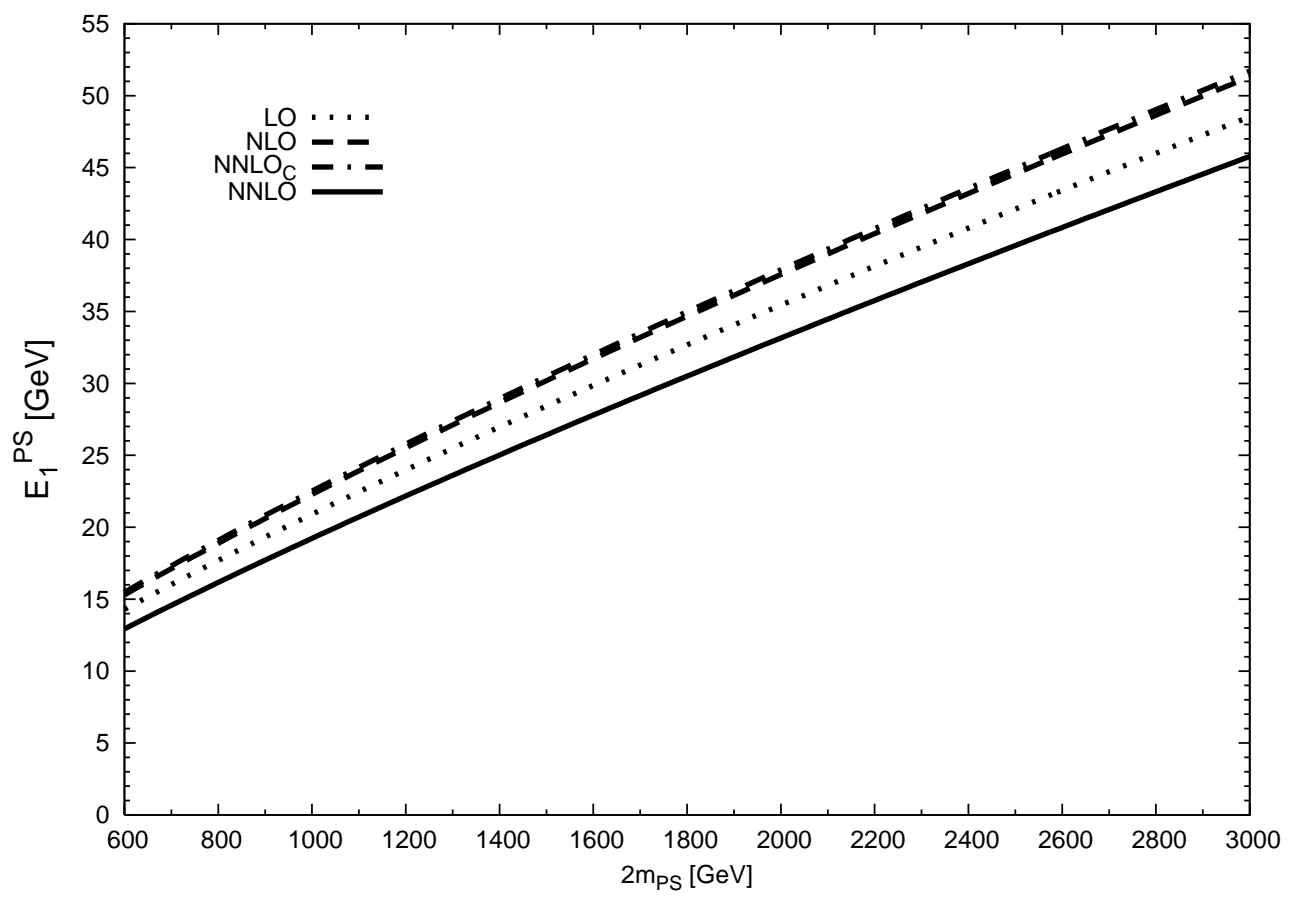

Figure 4: Ground state energy $E_{1}$ in the potential subtracted mass scheme as function of twice the potential subtracted mass. The curves have been obtained using the pole mass as input and evaluating both the potential subtracted mass (using Eq. (77) ) and $E_{1}^{P S}$ to a given order in $\alpha_{s}$. Note that the dash-dotted and dashed curves are almost on top of each other.

a poor convergence in both cases. The predictions for $M(2 S)-M(1 S)$ are shown in Fig. 5 , adopting $\mu=\mu_{S}$. The NNLO terms are evidently important and the poor convergence of the prediction for $E_{1}$ is reflected in this figure.

In the on-shell scheme the $1 S$ binding energy is evidently closely related to the energy difference between the groundstate and the onset of open gluino production or, more specifically, the threshold for pair production of color-neutral $(\tilde{g} g)$ hadrons. However, anticipating a mass resolution of several tens of $\mathrm{GeV}$ at least, it will be difficult to resolve the densely distributed radial excitations. For this reason the energy difference between the groundstate and the first radial excitation is a convenient measure of the isolation of the $1 S$ state.

Production and decay of non-relativistic $S$-wave bound states are proportional to the square of the wave function at the origin. The analytic results are collected in the Appendix A, numerical results are listed in Eq. (6) and shown in Fig. 6, The enormous size in particular of the non-Coulombic NNLO terms has also been observed for the top-antitop system and destabilizes the predictions [32]. At present the size of these corrections must be considered as an estimate of the theory uncertainty. 


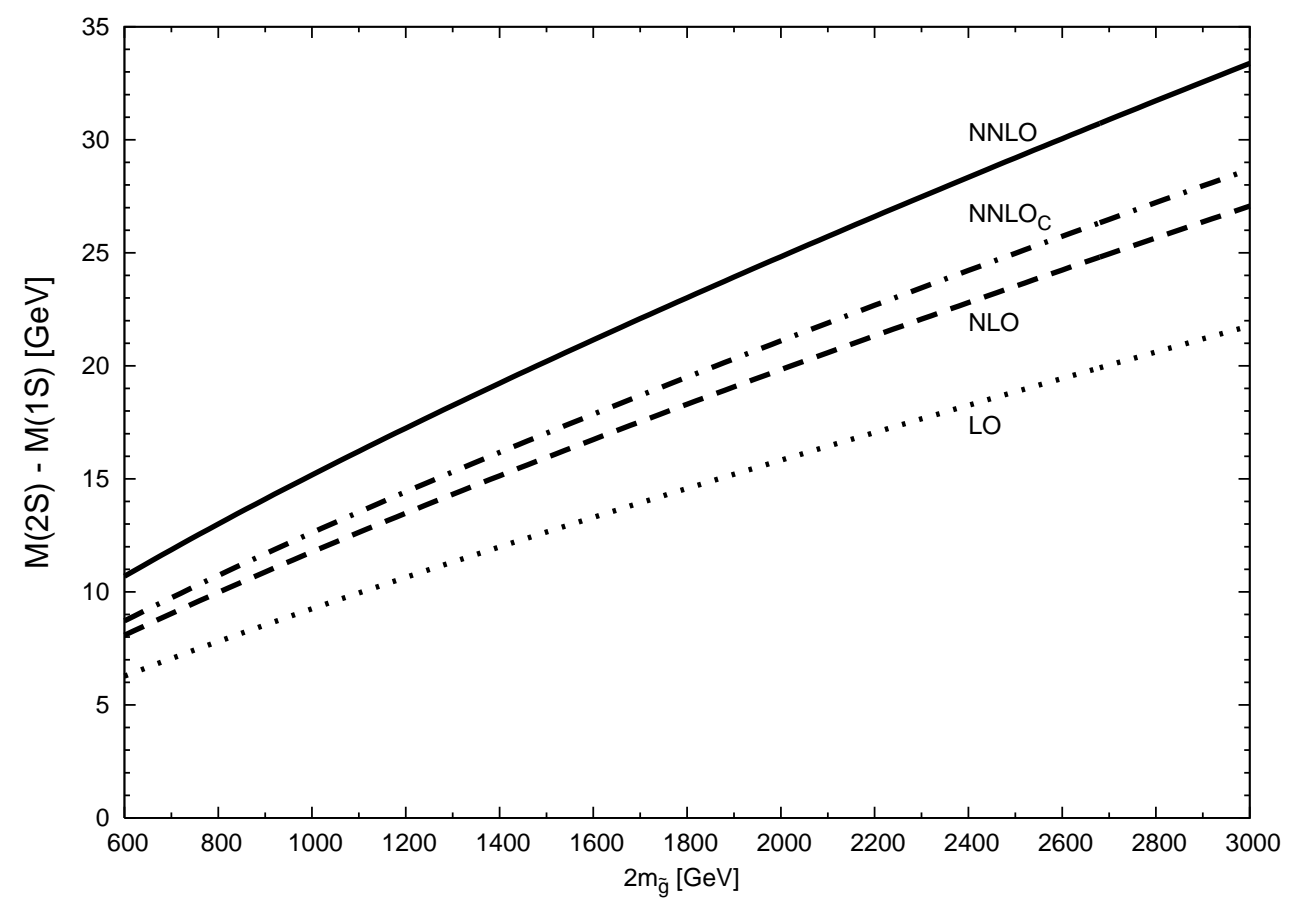

Figure 5: Meson mass difference $M(2 S)-M(1 S)$ as function of twice the constituent mass.

The renormalization scale dependence of the LO, NLO and $\mathrm{NNLO}_{C}$ results for $E_{1}$ and $\left|\Psi_{1}(0)\right|^{2}$ are shown in Figs. 7 and 8 for a constituent mass of $1 \mathrm{TeV}$. The predictions are normalized to the LO prediction evaluated at the scale $\mu$ as defined above. A significant stabilization is observed with increasing order in $\alpha_{s}$ and, furthermore, the higher order contributions are reasonably small for the preferred choice of the renormalization scale, at least, as far as the Coulombic part is concerned. This is in particular true for the ground state energy parameterized in terms of the potential subtracted mass where close to $\mu=150 \mathrm{GeV}$ the Coulombic NNLO results even vanish. In the case of the wave function one observes around $\mu=300 \mathrm{GeV}$ corrections of the order of a few percent from $\mathrm{NLO}$ and $\mathrm{NNLO}_{\mathrm{C}}$, however, a huge contribution from the non-Coulombic terms.

\section{Bound state production and decay}

In the present context squarks are assumed to be significantly heavier than gluinos, such that the direct decay $\tilde{g} \rightarrow \tilde{q} g$ is forbidden. An extreme example is provided by Split SUSY $[7,8]$ with $m_{\tilde{q}} \gg m_{\tilde{g}}$ and we shall adopt this simplifying mass assignment throughout this section. However, this assumption could be significantly relaxed. Indeed, once $m_{\tilde{q}}>m_{\tilde{g}}$ 


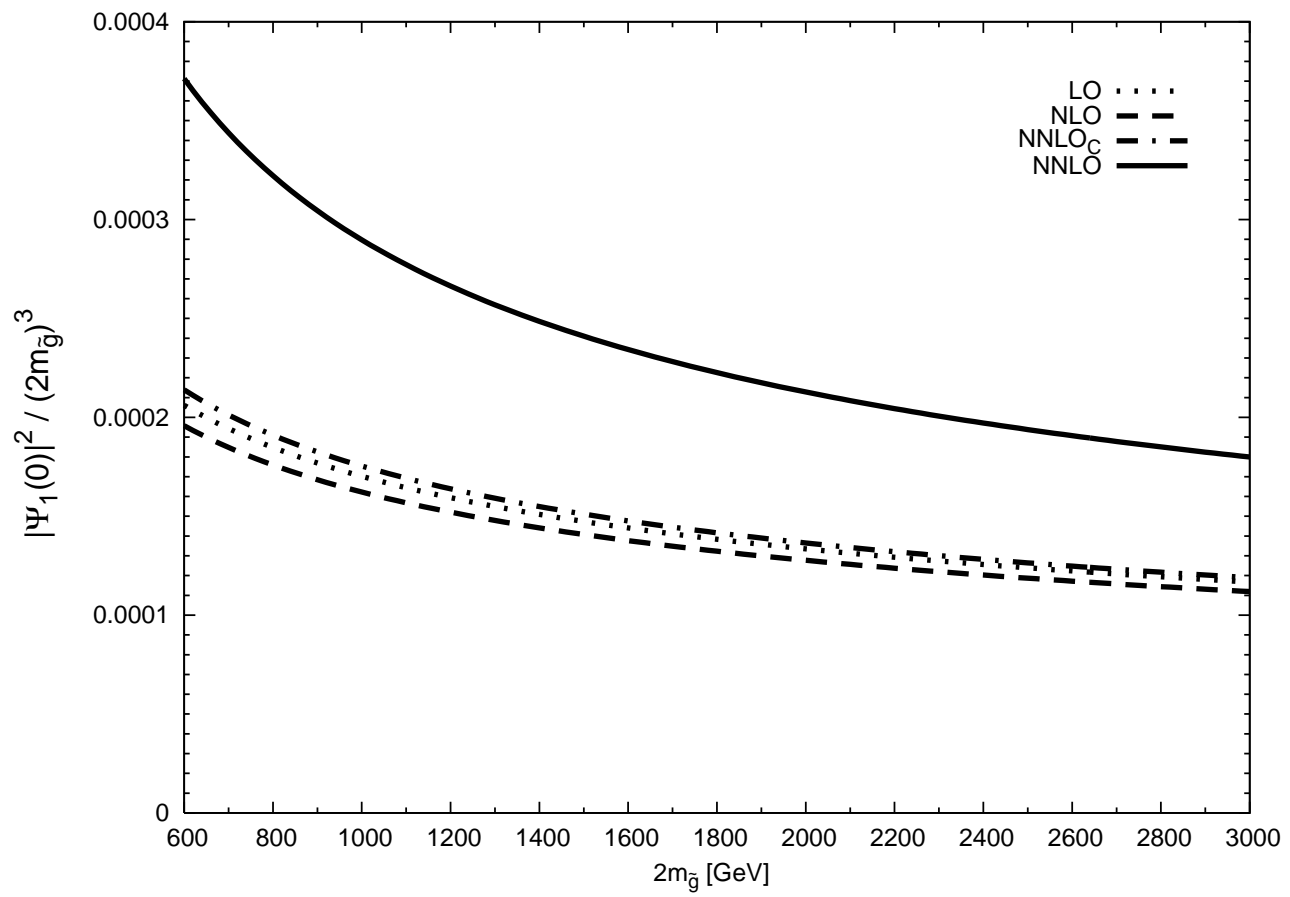

Figure 6: Squared ground state wave function at the origin as function of twice the constituent mass.

is valid the gluinos have a very small decay rate, for $m_{\tilde{q}} \gg m_{\tilde{g}}$ of order [42]

$$
\begin{aligned}
\Gamma(\tilde{g} \rightarrow \tilde{\gamma} q \bar{q}) & \approx\left(\sum_{q} e_{q}^{2}\right) \frac{\alpha \alpha_{s}}{48 \pi} \frac{m_{\tilde{g}}^{5}}{m_{\tilde{q}}^{4}} \\
& \approx 10 \mathrm{MeV}\left[\frac{m_{\tilde{g}}^{5}}{m_{\tilde{q}}^{4} \mathrm{TeV}}\right],
\end{aligned}
$$

characterizing the rate for gluino decays into neutralinos (similar estimates being applicable for decays into charginos) such that the decay rate is far smaller than the annihilation decay.

The evaluation of the rate for gluinonium annihilation into gluon (and quark) jets proceeds similar to the one for quarkonia. In Born approximation the color factor is replaced as follows

$$
\sum_{a, b}\left|\frac{\delta_{i k}}{\sqrt{N_{C}}} \frac{\lambda_{i j}^{a}}{2} \frac{\lambda_{j k}^{b}}{2}\right|^{2}=\frac{2}{3} \Rightarrow \sum_{a, b}\left|\frac{\delta_{i k}}{\sqrt{N_{C}^{2}-1}} f^{a i j} f^{b j k}\right|^{2}=9
$$

and another relative factor $\frac{1}{2}$ arises from the Majorana nature of the gluinos, whence [1-3]

$$
\Gamma_{\mathrm{LO}}\left(0^{-+} \rightarrow g g\right)=\Gamma_{g g}=\frac{C_{A}^{2}}{2} \frac{\alpha_{s}^{2}}{m_{\tilde{g}}^{2}}|R(0)|^{2}
$$



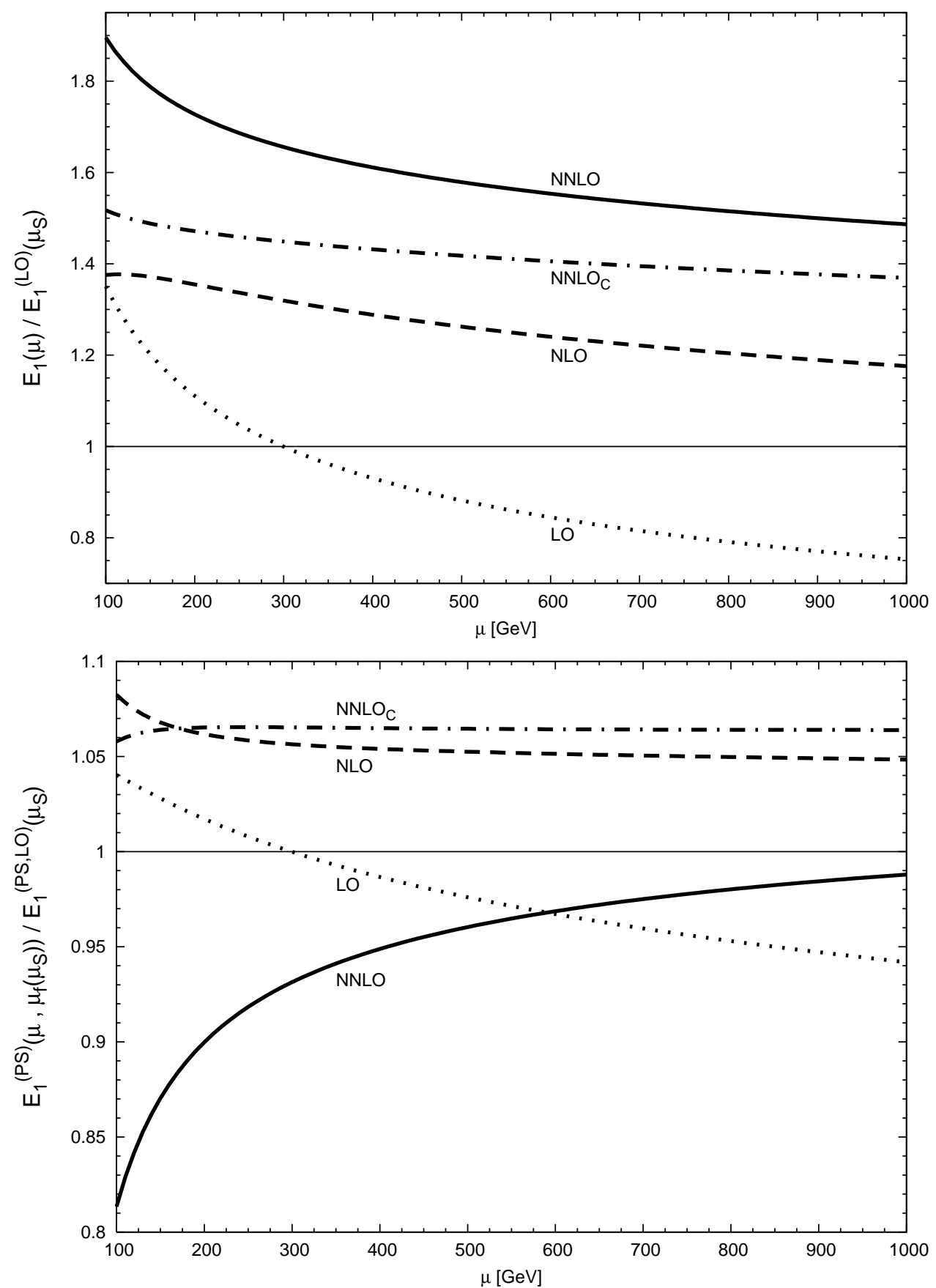

Figure 7: Renormalization scale dependence of the ground state energy for $m_{\tilde{g}}=1 \mathrm{TeV}$ in the pole mass scheme (a) and the potential subtracted scheme (b).

with $|R(0)|^{2}=4 \pi\left|\Psi_{1}(0)\right|^{2}$. Using the wave function obtained in lowest order perturbation theory leads to $\Gamma_{g g} \approx\left(C_{A} \alpha_{s}\right)^{5} m_{\tilde{g}} / 4$ which provides a qualitative estimate of the full result. The perturbative corrections arising from virtual and real emission can be calculated 


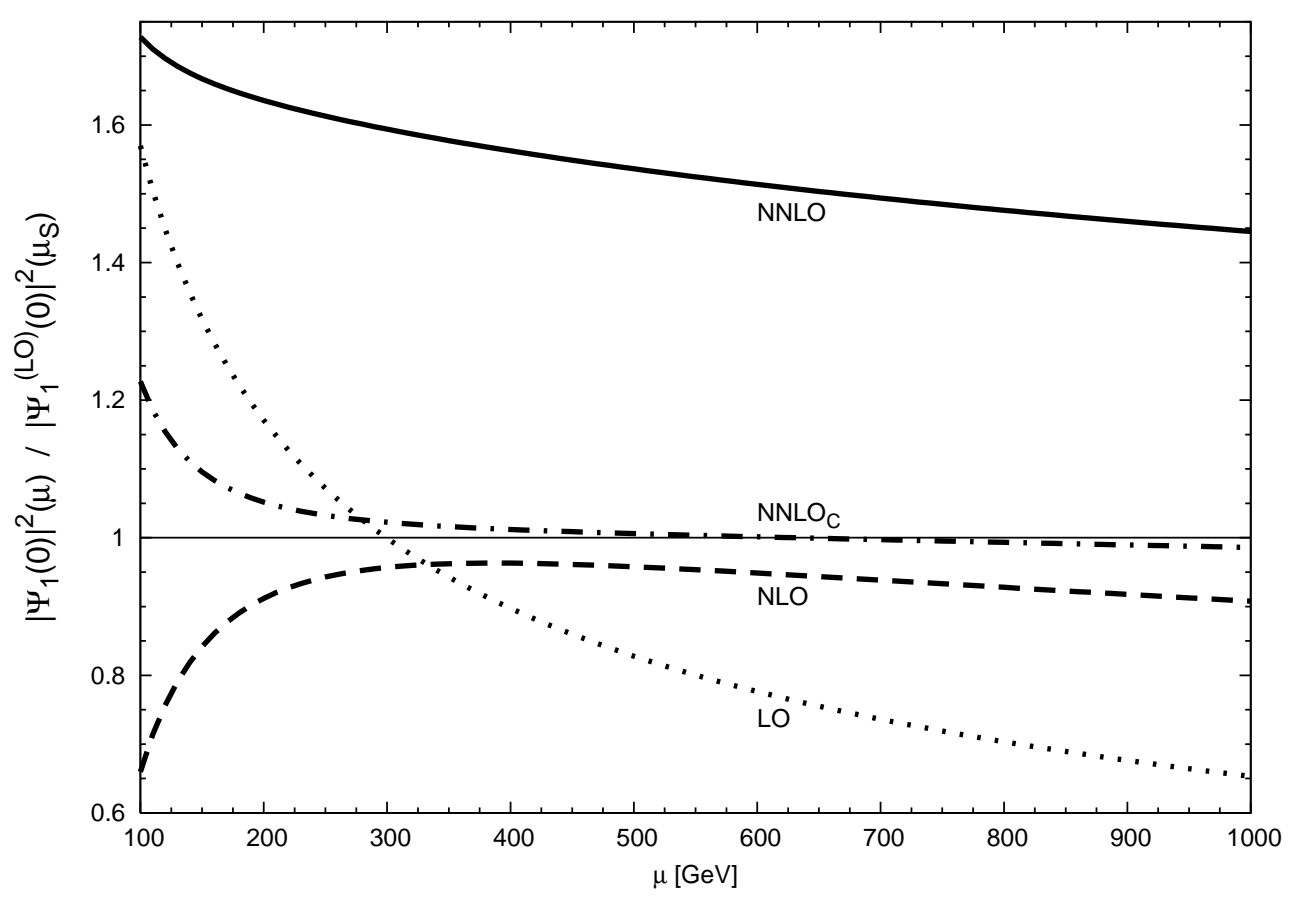

Figure 8: Renormalization scale dependence of the squared ground state wave function at the origin for $m_{\tilde{g}}=1 \mathrm{TeV}$.

similar to the ones for quarkonia [43]. They reduce the scale dependence of the lowest order approximation and are complementary to the higher order corrections to the wave function. In total one finds

$$
\begin{aligned}
\Gamma_{\mathrm{NLO}}= & \Gamma_{g g}\left\{1+\frac{\alpha_{s}(\mu)}{\pi}\left[C_{A}\left(\frac{109}{18}-\frac{7}{24} \pi^{2}\right)-\frac{16}{9} n_{f} T_{F}\right.\right. \\
& \left.\left.+\left(\frac{11}{6} C_{A}-\frac{2}{3} n_{f} T_{F}\right) \ln \left(\frac{\mu^{2}}{4 m_{\tilde{g}}^{2}}\right)\right]\right\} .
\end{aligned}
$$

Here $\alpha_{s}$ is understood in the theory with $n_{f}=6$ effective flavors and all quark masses, including $m_{t}$, have been neglected. The $\alpha_{s}^{3}$ term present in $|R(0)|^{2}$ is interpreted in the $n_{f}=5$ effective theory. The predictions for $\Gamma_{\mathrm{LO}}$ and $\Gamma_{\mathrm{NLO}}$ are shown in Fig. 9, where the NNLO wave function as shown in Fig. [6 (excluding the non-Coulombic contribution) and $\mu=2 m_{\tilde{g}}$ for the renormalization scale in Eq. (11) have been adopted. As anticipated, the decay rate of the bound state is small compared to the level spacing and large compared to $\Gamma(\tilde{g} \rightarrow \tilde{\gamma} q \bar{q})$, the decay rate of a single gluino, once $m_{\tilde{g}}<m_{\tilde{q}}$. Hence annihilation decays will constitute the dominant signal. However, it should be emphasized, that bound states will exist even for $m_{\tilde{g}}>m_{\tilde{q}}$, as long as $\Gamma(\tilde{g} \rightarrow \tilde{q} q) \leq E_{2}-E_{1}$, which is true for the region $m_{\tilde{q}} \gtrsim 0.9 m_{\tilde{g}}$. 


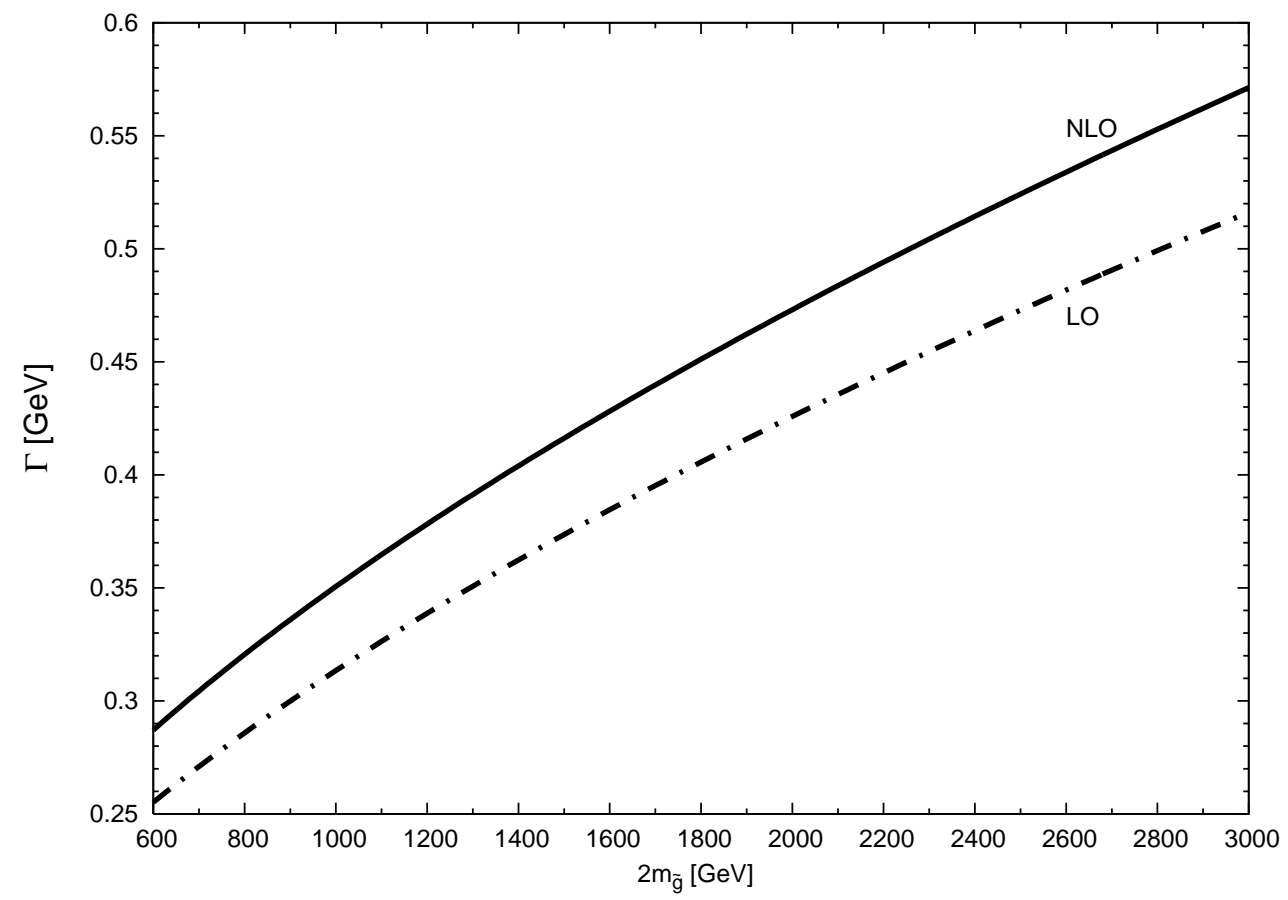

Figure 9: Decay rate of the $1 S$ state into gluons.

The bound state production cross section can be calculated similarly to the one for the pseudoscalar bound state of two top quarks (usually called $\eta_{t}$ ) [43]. In Born approximation the reaction proceeds through gluon fusion and the radiative corrections involve additional $g q, g \bar{q}$ and $q \bar{q}$ initiated subprocesses. The final result is quite similar to the one for quarkonia production [43]. We find

$$
\begin{aligned}
\sigma_{\text {had }}(S)= & \sum_{a b} \int_{0}^{1} \mathrm{~d} x_{1} \int_{0}^{1} \mathrm{~d} x_{2} f_{a}^{h_{1}}\left(x_{1}, \mu_{F}^{2}\right) f_{b}^{h_{2}}\left(x_{2}, \mu_{F}^{2}\right) \hat{\sigma}_{a b}\left(s=x_{1} x_{2} S\right), \\
\hat{\sigma}_{g g}= & \sigma_{0}\left\{\delta(1-z)+\frac{\alpha_{s}}{\pi}\left[-P_{g g}(z) \ln \left(\frac{\mu^{2}}{4 m_{\tilde{g}}^{2}}\right)+C_{A} F(z)\right.\right. \\
& \left.\left.+\delta(1-z)\left(\left(\frac{11}{6} C_{A}-\frac{2}{3} n_{f} T_{F}\right) \ln \left(\frac{\mu^{2}}{4 m_{\tilde{g}}^{2}}\right)+C_{A}\left(-4+\frac{1}{3} \pi^{2}\right)\right)\right]\right\}, \\
\hat{\sigma}_{g q}= & \sigma_{0} \frac{\alpha_{s}}{\pi}\left\{-C_{F} \frac{z}{2} \ln z+C_{F} z+\frac{1}{2} P_{g q}(z)\left(\ln \left(\frac{4 m_{\tilde{g}}^{2}(1-z)^{2}}{\mu^{2}}\right)-1\right)\right\}, \\
\hat{\sigma}_{g \bar{q}}= & \hat{\sigma}_{g q}, \\
\hat{\sigma}_{q \bar{q}}= & \sigma_{0} \frac{\alpha_{s}}{\pi} \frac{32}{27} z(1-z),
\end{aligned}
$$


with

$$
\begin{aligned}
\sigma_{0}= & \frac{C_{A}^{2} \pi^{2} \alpha_{s}^{2}}{4} \frac{|R(0)|^{2}}{s\left(2 m_{\tilde{g}}\right)^{3}}, \\
P_{g g}(z)= & 2 C_{A}\left(\frac{1}{z}+\left[\frac{1}{1-z}\right]_{+}+z(1-z)-2\right) \\
& +\left(\frac{11}{6} C_{A}-\frac{2}{3} n_{f} T_{F}\right) \delta(1-z), \\
P_{g q}(z)= & C_{F} \frac{1+(1-z)^{2}}{z}, \\
F(z)= & \frac{11 z^{5}+11 z^{4}+13 z^{3}+19 z^{2}+6 z-12}{6 z(1+z)^{2}} \\
& +4\left(\frac{1}{z}+z(1-z)-2\right) \ln (1-z)+4\left[\frac{\ln (1-z)}{1-z}\right]_{+} \\
& +\left(\frac{2\left(z^{3}-2 z^{2}-3 z-2\right)\left(z^{3}-z+2\right) z \ln z}{(1+z)^{3}(1-z)}-3\right) \frac{1}{1-z} .
\end{aligned}
$$

Here $z=4 m_{\tilde{g}}^{2} / s \leq 1, \mu$ and $\mu_{F}$ are the renormalization and the factorization scales, $\alpha_{s}$ is again defined in the $n_{f}=6$ flavor theory, $P_{i j}$ are the Altarelli-Parisi splitting functions and $[\ldots]_{+}$denotes the usual + distribution. For the wave function at the origin we again adopt the NNLO result without the non-Coulombic terms. The predictions for the production cross section are shown in Fig. 10 where the parton distribution functions MSTW2008LO (MSTW2008NLO) [44] have been used for the LO (NLO) calculation. Leading order (dashed) and NLO (solid curve) predictions are evaluated using $\mu=\mu_{F}=2 m_{\tilde{g}}$.

For a gluino mass of $1 \mathrm{TeV}$ the $\mu$ dependence (with $\mu_{F}=\mu$ ) of the LO and the NLO results is shown in Fig. 11, together with the decomposition of the NLO result into the contributions from the different subprocesses.

From Figs. 10 and 11 we learn that the NLO correction amounts to more than $+70 \%$ fairly independent of the renormalization scale. The dominant uncertainty, not displayed in these figures, arises from the wave function at the origin, with indications for an increase by another factor of two.

For an anticipated integrated luminosity of $100 \mathrm{fb}^{-1}$ between 10 and $10^{6}$ events are expected for $m_{\tilde{g}}$ between $1.5 \mathrm{TeV}$ and $300 \mathrm{GeV}$. It remains to be seen, if these can be separated from the hadronic continuum background.

\section{Annihilation decays into top quarks and into two photons}

In the limit of extremely heavy squarks (and conserved R-parity), corresponding to Split SUSY, decay modes of single gluinos are extremely suppressed, such that gluinos (con- 


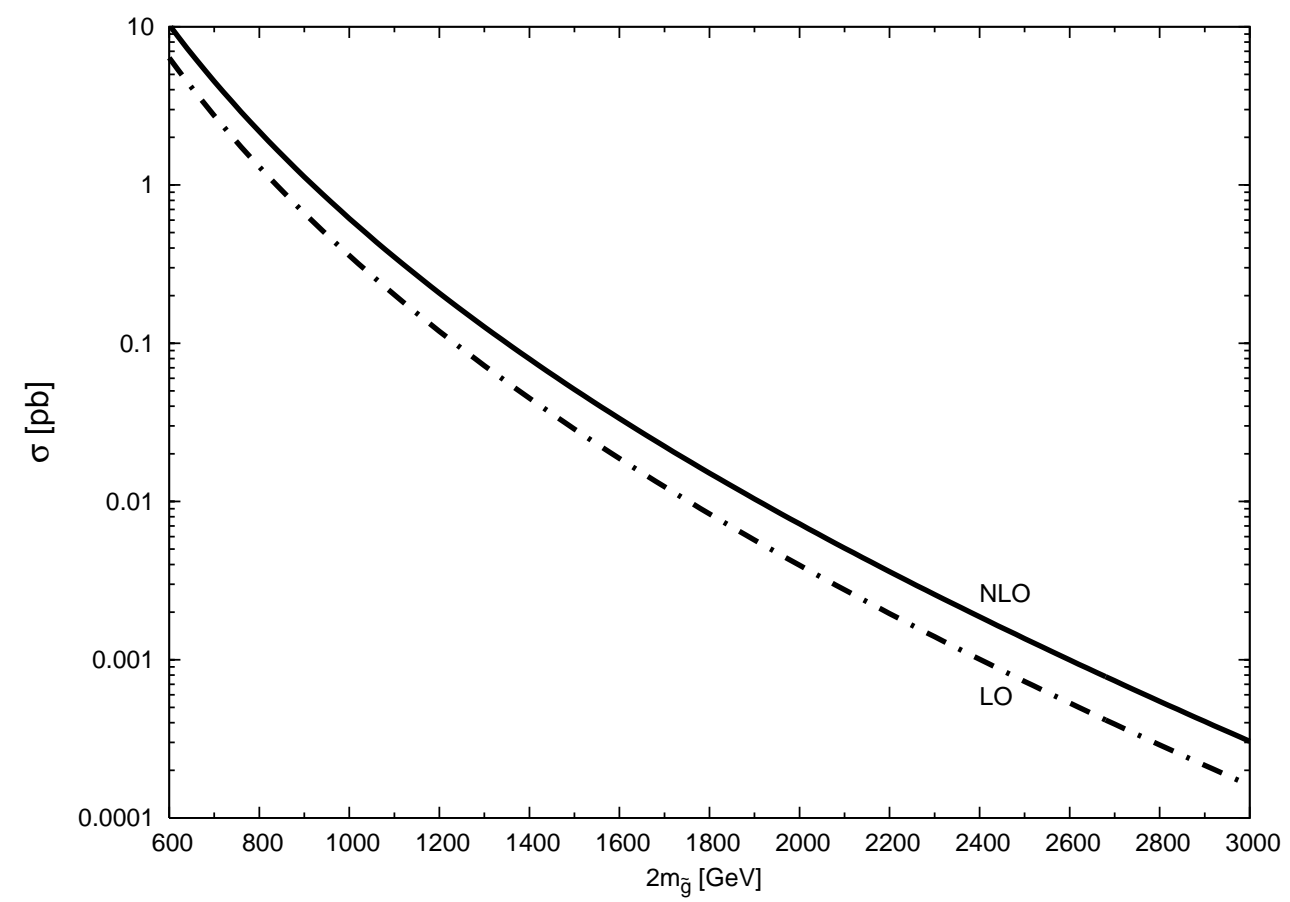

Figure 10: LO and NLO production cross section for the $1 S$ state at $\sqrt{s}=14 \mathrm{TeV}$.

fined in color-neutral hadrons like $\tilde{g} g$ ) could even travel microscopic distances. In this case gluinonium annihilation into two gluons is the only relevant channel. However, once squark and gluino masses are comparable, new annihilation decays become possible. At first glance one might consider the mode $(\tilde{g} \tilde{g}) \rightarrow q \bar{q}$, mediated by squark exchange. However, for massless quarks this process vanishes as a consequence of helicity conservation. For massive top quarks one finds

$$
R_{t \bar{t}}=\frac{\Gamma\left(0^{-+} \rightarrow t \bar{t}\right)}{\Gamma\left(0^{-+} \rightarrow g g\right)}=\frac{4 C_{F}}{C_{A}^{2}} \sqrt{1-\frac{m_{t}^{2}}{m_{\tilde{g}}^{2}}} \frac{m_{\tilde{g}}^{2} m_{t}^{2}}{\left(m_{\tilde{g}}^{2}+m_{\tilde{t}}^{2}-m_{t}^{2}\right)^{2}} .
$$

using $m_{\tilde{t}}=m_{\tilde{t}_{1}}=m_{\tilde{t}_{2}}$. The complete result for $m_{\tilde{t}_{1}} \neq m_{\tilde{t}_{2}}$ and including squark mixing is given in Appendix B.1.

The result is plotted in Fig. 12 for different ratios of $m_{\tilde{t}} / m_{\tilde{g}}$. Branching ratios of up to $7 \%$ are possible for gluino masses close to $300 \mathrm{GeV}$ and squark masses not much bigger. For heavier squark and gluino masses the ratio drops quickly.

As a second possibility we consider the loop induced decay into two photons. Although suppressed by the electromagnetic couplings $\alpha^{2}$, the cleaner signal-to-background ratio might (at least in principle) compensate this disadvantage. For arbitrary squark mixing and masses the result is given in Appendix B.2. In the limit of massless quarks and 


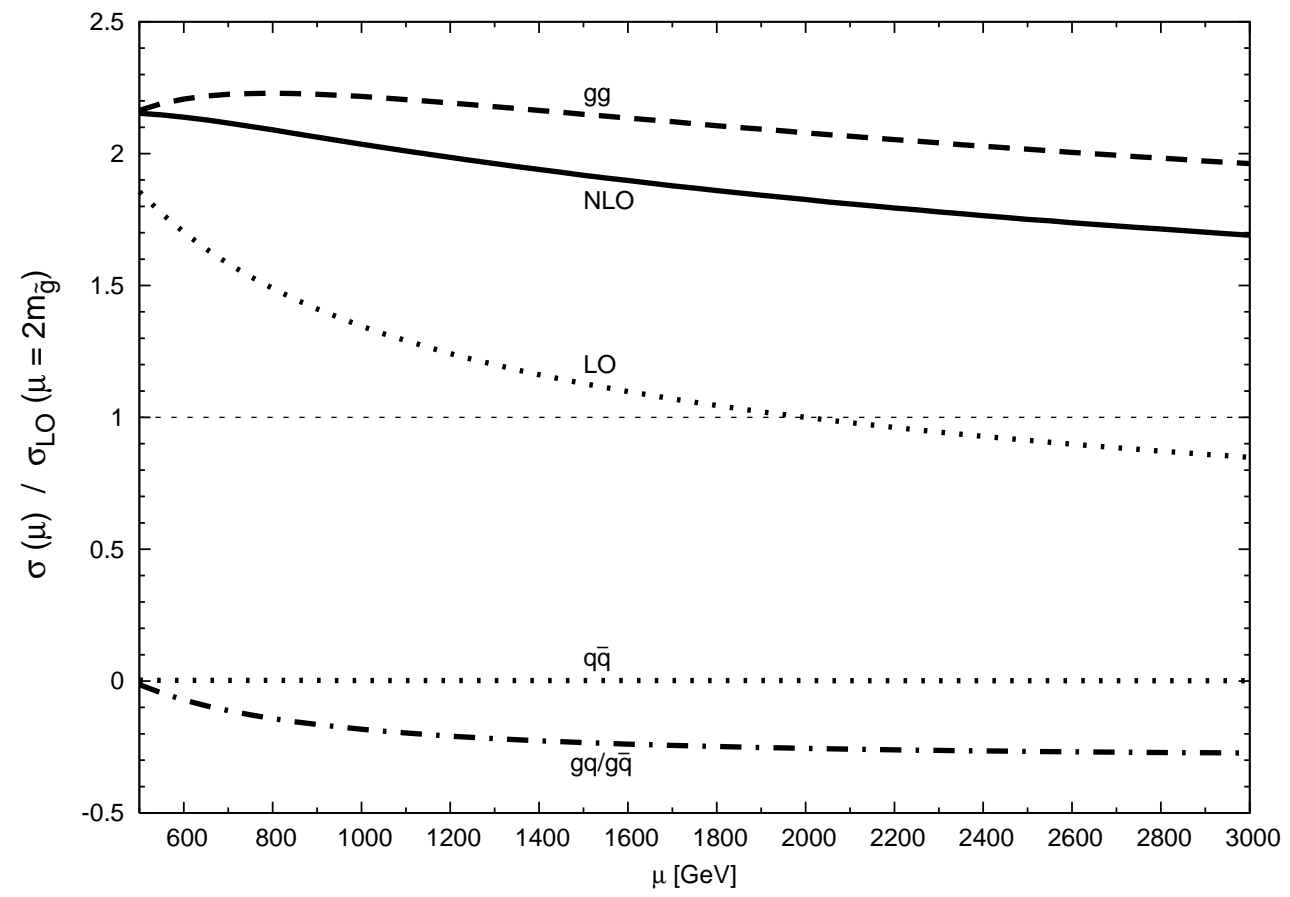

Figure 11: Renormalization- and factorization-scale dependence of the $1 S$ production cross section and decomposition into contributions from various subprocesses. The line labeled "gg" contains also the LO result.

degenerate squarks the decay rate simplifies considerably

$$
R_{\gamma \gamma}=\frac{\Gamma\left(0^{-+} \rightarrow \gamma \gamma\right)}{\Gamma\left(0^{-+} \rightarrow g g\right)}=\frac{4 T_{F}}{C_{A}^{2}} \frac{\alpha^{2}}{\pi^{2}}\left(\sum_{f} Q_{f}^{2}\right)^{2}\left|\operatorname{Li}_{2}\left(-\frac{m_{\tilde{g}}^{2}}{m_{\tilde{q}}^{2}}\right)-\operatorname{Li}_{2}\left(\frac{m_{\tilde{g}}^{2}}{m_{\tilde{q}}^{2}}\right)\right|^{2}
$$

With a ratio $R_{\gamma \gamma}$ around one to several $10^{-5}$ the two-photon signal will not be detectable.

\section{Signal versus background}

Assuming the two-gluon mode to dominate the decay of gluino bound states, the resonant signal must be isolated from the two-jet continuum. Below we present an order of magnitude estimate for the signal-to-background ratio, following essentially the arguments given in Ref. [3]. For simplicity it is assumed that quark and gluon jets can be experimentally separated. In this case the background is dominated by gluon-gluon scattering. Furthermore, we enhance the signal-to-background ratio by excluding small scattering angles in the partonic center-of-mass system, requiring $|\cos (\theta)| \leq z<1$.

To isolate a resonance in the gluon-gluon channel, a good experimental resolution $\Delta M$ of the invariant dijet mass $M_{j j}$ is essential. Let us compare the differential cross section, 


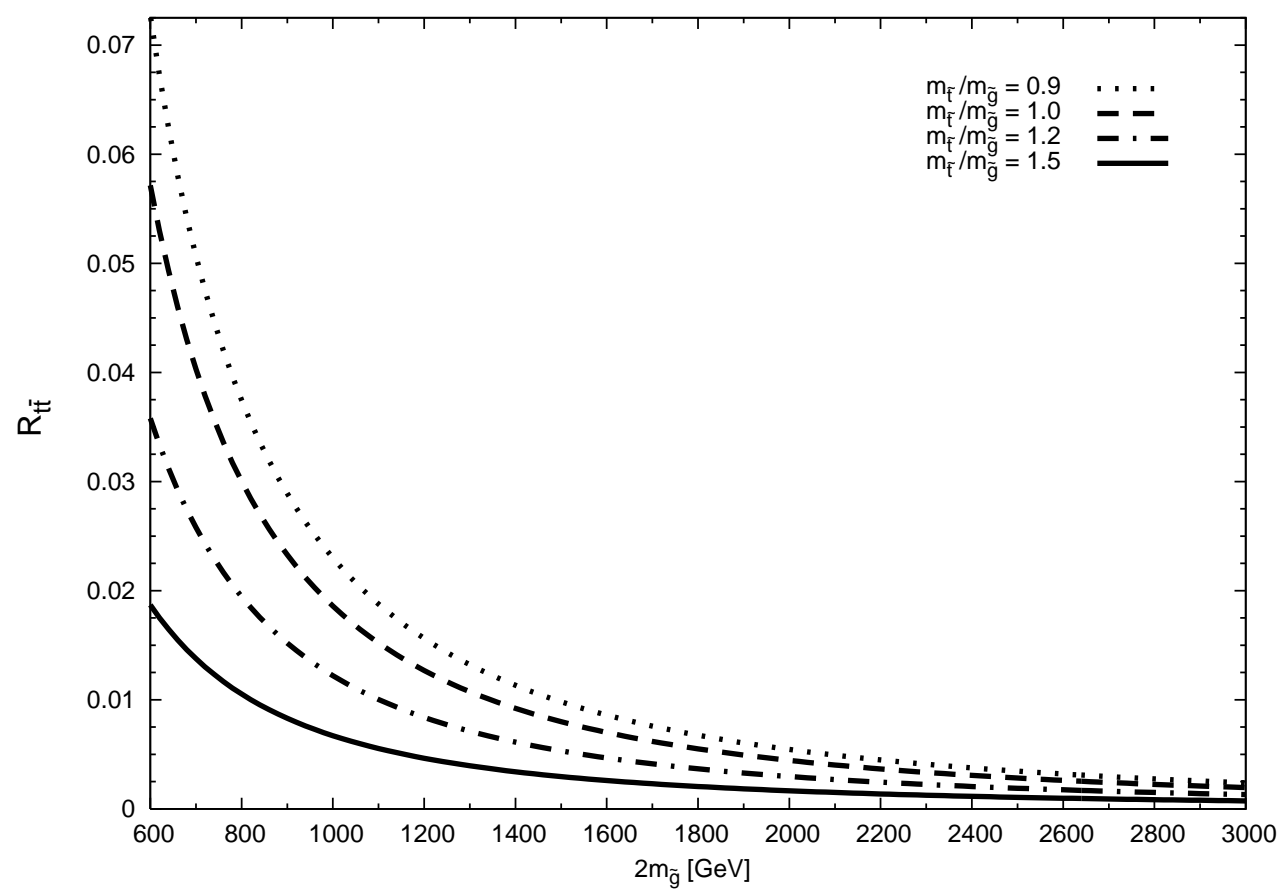

Figure 12: Branching ratio of the $1 S$ decay rate into top quarks.

integrated over the interval $M-\frac{\Delta M}{2} \leq M_{j j} \leq M+\frac{\Delta M}{2}$ for background and signal, and integrated over the same angular range. This leads to the signal-to-background ratio

$$
r(z, \Delta M)=\frac{S(z, \Delta M)}{B(z, \Delta M)}=\frac{\pi}{3} \frac{\Gamma_{g g}}{\Delta M \alpha_{s}^{2}}\left[\frac{129-32 z^{2}-z^{4}}{1-z^{2}}-\frac{24}{z} \log \left(\frac{1+z}{1-z}\right)\right]^{-1} .
$$

Using $\Delta M / M_{j j}=0.038+38 / M_{j j}$ for the dijet resolution [45], one obtains the signal-tobackground ratio as shown in Fig. 13, varying between $0.4 \%$ to $0.7 \%$. Given event rates between $10^{3}$ and $10^{5}$ for $m_{\tilde{g}}$ between $500 \mathrm{GeV}$ and $1 \mathrm{TeV}$ the detection of gluino bound states might become feasible, in particular at a high luminosity version of the LHC.

\section{Conclusions}

For a large class of supersymmetric extensions of the Standard Model gluinos will be sufficiently stable to form color-neutral non-relativistic boundstates. The NNLO potential between color-octet constituents forming color singlets has been derived. Just as in leading and next-to-leading order the Coulombic part of the NNLO potential is related to the quarkonium potential through the substitution $C_{F} \rightarrow C_{A}$ in the overall factor. The transformation of the short distance terms involving an additional factor $1 / m$ is only slightly more complicated and discussed in Section 2. Based on this potential, predictions 


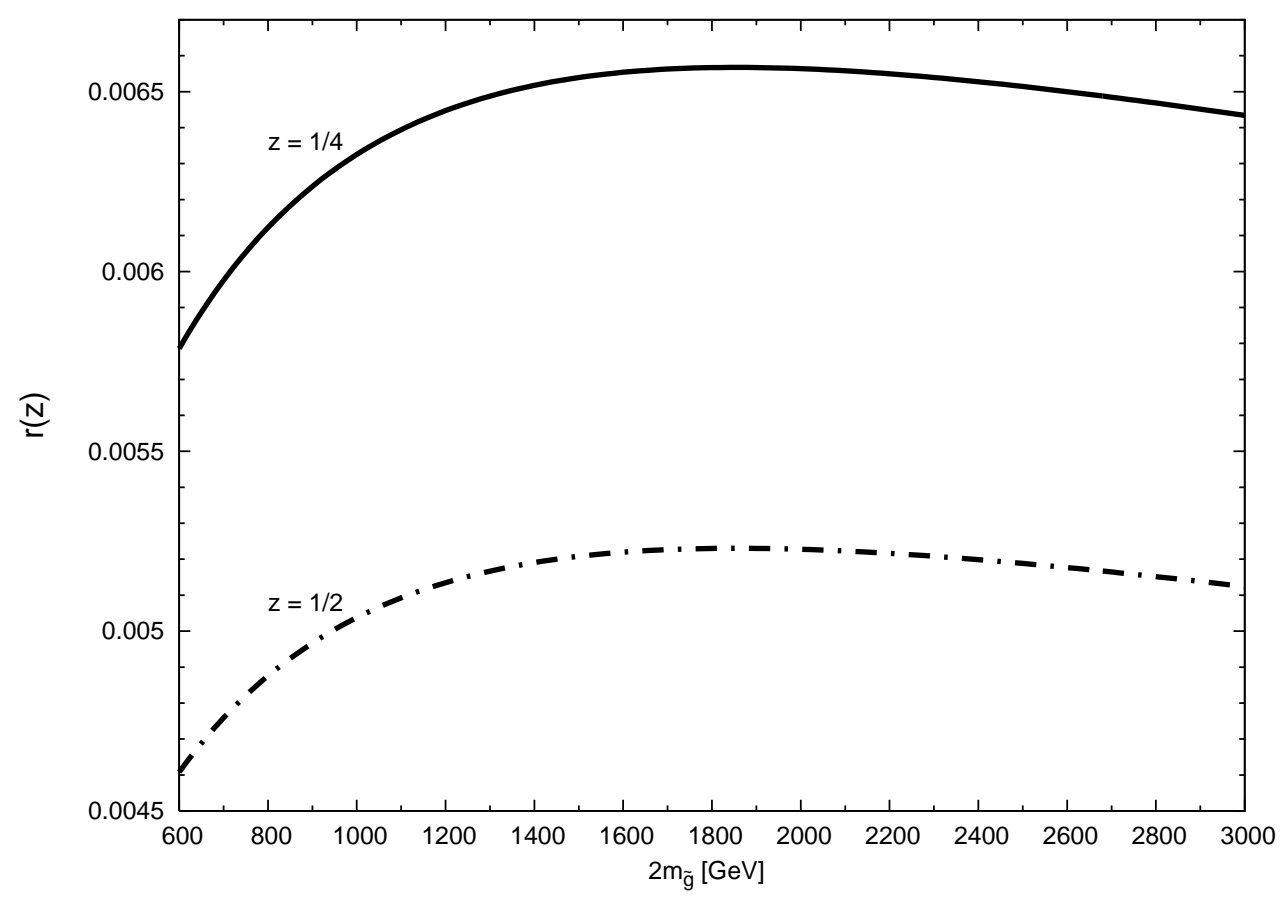

Figure 13: Signal-to-background ratio for different values of $z$.

for the binding energy and the wave function at the origin of the lowest-lying boundstates and the first radial excitation have been derived. For gluino masses between $300 \mathrm{GeV}$ and $1500 \mathrm{GeV}$ the $1 \mathrm{~S}$ binding energy (in the pole mass scheme) varies between $15 \mathrm{GeV}$ and $50 \mathrm{GeV}$, the $1 S-2 S$ energy difference between $10 \mathrm{GeV}$ and $30 \mathrm{GeV}$. For the case of gluinos being lighter than squarks the gluino decay rate is significantly smaller than the level spacing and the boundstates are thus well separated from the continuum. Also the wave function at the origin, which enters bound state production and decay rates, has been evaluated in NNLO. For $m_{\tilde{g}}>m_{\tilde{q}}$ the decay rate of gluinonia into two gluon jets is evaluated in NLO and shown to dominate all other modes. For top squarks with masses comparable to $m_{\tilde{g}}$ the decay mode into $t \bar{t}$ may reach several percent, the decay through virtual quark loops into two photons is too small to be detectable. We evaluate the boundstate production cross section again in NLO. Large positive corrections of around $50 \%$ arise from the NNLO terms in the wave function at the origin and around $70 \%$ from the NLO terms in the cross section. The production rate depends strongly on the gluino mass, with up to $10^{6}$ events for gluino masses close to the lower limit around $300 \mathrm{GeV}$ (for an integrated luminosity of $100 \mathrm{fb}^{-1}$ ) dropping quickly to 10 events only for $m_{\tilde{g}}=1.5 \mathrm{TeV}$. Using standard assumptions for the dijet-mass resolution and assuming that gluon and quark jets can be separated, a signal-to-background ratio between 0.4 and $0.7 \%$ percent might be conceivable. For favorable gluino- and squark-mass combinations gluinonia might thus be detectable. 
Acknowledgements M.S. thanks Alexander Penin for discussions about $f_{2}^{n C}$. This work was supported by the BMBF through Grant No. 05H09VKE.

Note added: After completion of this work we received a preprint by K. Hagiwara and H. Yokoya where the production of unstable gluinos in the threshold region is considered [46].

\section{A Energy levels and wave functions}

For completeness we present the perturbative analytic results for the energy levels $E_{n}$ of the $S$ waves and the corresponding values of the squared wave function at the origin $\left|\Psi_{n}(0)\right|^{2}$, truncating the perturbative series at NNLO. We present the results following the notation of [39]. We define

$$
\begin{aligned}
E_{n} & =E_{n}^{(0)}\left[1+\frac{\alpha_{s}}{4 \pi} e_{1}+\left(\frac{\alpha_{s}}{4 \pi}\right)^{2} e_{2}\right] \\
\left|\Psi_{n}(0)\right|^{2} & =\left|\Psi_{n}^{(0)}(0)\right|^{2}\left[1+\frac{\alpha_{s}}{4 \pi} f_{1}+\left(\frac{\alpha_{s}}{4 \pi}\right)^{2} f_{2}\right]
\end{aligned}
$$

and parameterize the corrections as

$$
\begin{aligned}
e_{i} & =e_{i}^{C}+e_{i}^{n C} \\
f_{i} & =f_{i}^{C}+f_{i}^{n C}
\end{aligned}
$$

where $C$ stands for the corrections to the Coulombic part of the potential and $n C$ for the remaining one. The $\mathrm{LO}$ values are given as

$$
\begin{aligned}
E_{n}^{(0)} & =-\frac{m_{\tilde{g}} C_{A}^{2} \alpha_{s}^{2}}{4 n^{2}}, \\
\left|\Psi_{n}^{(0)}(0)\right|^{2} & =\frac{m_{\tilde{g}}^{3} C_{A}^{3} \alpha_{s}^{3}}{8 \pi n^{3}} .
\end{aligned}
$$


The NLO and NNLO corrections are

$$
\begin{aligned}
& e_{1}^{C}=4 \beta_{0} \ln \left(\frac{n \mu}{m_{\tilde{g}} C_{A} \alpha_{s}}\right)+2 a_{1}+4 S_{1} \beta_{0}, \\
& e_{1}^{n C}=0 \\
& e_{2}^{C}=12 \beta_{0}^{2} \ln ^{2}\left(\frac{n \mu}{m_{\tilde{g}} C_{A} \alpha_{s}}\right)+\ln \left(\frac{n \mu}{m_{\tilde{g}} C_{A} \alpha_{s}}\right)\left[-8 \beta_{0}^{2}+4 \beta_{1}+6 \beta_{0}\left(2 a_{1}+4 S_{1} \beta_{0}\right)\right] \\
& +a_{1}^{2}+2 a_{2}+4 S_{1} \beta_{1}+4 a_{1} \beta_{0}\left(3 S_{1}-1\right) \\
& +\beta_{0}^{2}\left[S_{1}\left(12 S_{1}-8-\frac{8}{n}\right)+16 S_{2}-8 n S_{3}+\frac{2 \pi^{2}}{3}+8 n \zeta(3)\right], \\
& e_{2}^{n C}=\frac{16 \pi^{2} C_{A}^{2}}{n}\left(3-\frac{11}{16 n}-\frac{2}{3} \vec{S}^{2}\right) \\
& f_{1}^{C}=6 \beta_{0} \ln \left(\frac{n \mu}{m_{\tilde{g}} C_{A} \alpha_{s}}\right)+3 a_{1}+2 \beta_{0}\left(S_{1}+2 n S_{2}-1-\frac{n \pi^{2}}{3}\right), \\
& f_{1}^{n C}=0, \\
& f_{2}^{C}=24 \beta_{0}^{2} \ln ^{2}\left(\frac{n \mu}{m_{\tilde{g}} C_{A} \alpha_{s}}\right) \\
& +\ln \left(\frac{n \mu}{m_{\tilde{g}} C_{A} \alpha_{s}}\right)\left\{-12 \beta_{0}^{2}+6 \beta_{1}+8 \beta_{0}\left[3 a_{1}+2 \beta_{0}\left(S_{1}+2 n S_{2}-1-\frac{n \pi^{2}}{3}\right)\right]\right\} \\
& +3 a_{1}^{2}+3 a_{2}+2 a_{1} \beta_{0}\left(4 S_{1}+8 n S_{2}-7-\frac{4 n \pi^{2}}{3}\right)+2 \beta_{1}\left(S_{1}+2 n S_{2}-1-\frac{n \pi^{2}}{3}\right) \\
& +\beta_{0}^{2}\left[S_{1}\left(8 S_{1}+16 n S_{2}-20-\frac{12}{n}-\frac{8 n \pi^{2}}{3}\right)+S_{2}\left(4 n^{2} S_{2}+8-8 n-\frac{4 n^{2} \pi^{2}}{3}\right)\right. \\
& \left.+28 n S_{3}-20 n^{2} S_{4}-24 n S_{2,1}+16 n^{2} S_{3,1}+4+\frac{(3+4 n) \pi^{2}}{3}+\frac{n^{2} \pi^{4}}{9}+20 n \zeta(3)\right], \\
& f_{2}^{n C}=16 \pi^{2} C_{A}^{2}\left\{3 \ln \left(\frac{n \mu}{m_{\tilde{g}} C_{A} \alpha_{s}}\right)-3 S_{1}+\frac{6}{n}-\frac{15}{8 n^{2}}+\frac{21}{4}\right. \\
& \left.+\vec{S}^{2}\left[-\frac{2}{3} \ln \left(\frac{n \mu}{m_{\tilde{g}} C_{A} \alpha_{s}}\right)+\frac{2}{3} S_{1}-\frac{4}{3 n}-\frac{7}{9}\right]\right\} \text {. }
\end{aligned}
$$

The constants $a_{1}, a_{2}, \beta_{0}$ and $\beta_{1}$ depend on the Casimir operators $C_{A}, C_{F}$ and $T_{F}$ and are defined in Eq. (5). The harmonic sums $S_{i}(n)$, the nested harmonic sums $S_{i, j}(n)$ and the zeta-function $\zeta(i)$ are given as

$$
\begin{aligned}
S_{i}=S_{i}(n) & =\sum_{k=1}^{n} \frac{1}{k^{i}}, \\
S_{i, j}=S_{i, j}(n) & =\sum_{k=1}^{n} \frac{1}{k^{i}} S_{j}(k), \\
\zeta(i) & =\sum_{k=1}^{\infty} \frac{1}{k^{i}} .
\end{aligned}
$$



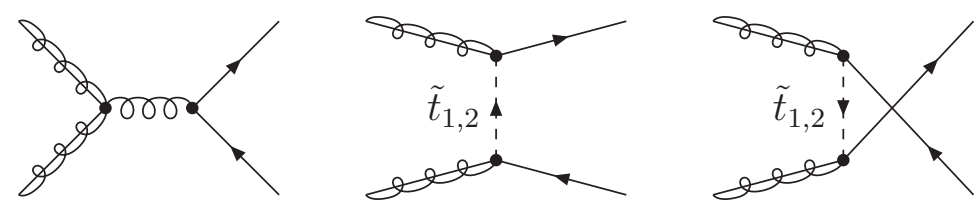

Figure 14: Feynman diagrams for the decay of $(\tilde{g} \tilde{g})$ into $t \bar{t}$.

\section{B Amplitudes for the decays into top quarks and into photons}

\section{B.1 Decay into top quarks}

In Fig. 14 the corresponding diagrams for the decay of two gluinos into top-antitop are shown. Diagram $(\alpha)$ drops out for the case of interest because the two gluinos are projected here onto a color-singlet state. The resulting amplitude for $(\beta)$ and $(\gamma)$ reads

$$
\begin{aligned}
\left.\mathcal{A}\right|_{\text {color singlet }}= & \sum_{h=1}^{2} \frac{C_{F} \delta_{i j}}{\sqrt{N_{C}^{2}-1}} \frac{2 i g_{s}^{2}}{m_{\tilde{g}}^{2}+m_{\tilde{t}_{h}}^{2}-m_{t}^{2}} \bar{u}_{s_{1}}\left(k_{1}, m_{t}\right)\left(P_{-} U_{h 2}^{(\tilde{t})}-P_{+} U_{h 1}^{(\tilde{t})}\right) \\
& \times\left[u_{\bar{s}}\left(\frac{P}{2}, m_{\tilde{g}}\right) \bar{v}_{s}\left(\frac{P}{2}, m_{\tilde{g}}\right)\right. \\
& \left.-u_{s}\left(\frac{P}{2}, m_{\tilde{g}}\right) \bar{v}_{\bar{s}}\left(\frac{P}{2}, m_{\tilde{g}}\right)\right]\left(P_{-} U_{h 1}^{(\tilde{t})}-P_{+} U_{h 2}^{(\tilde{t})}\right) v_{s_{2}}\left(k_{2}, m_{t}\right),
\end{aligned}
$$

where $s_{1}, s_{2}, s$ and $\bar{s}$ are the spin indices of the top, the anti-top and of the gluinos, $P_{-}$ and $P_{+}$are the left- and right-handed projectors as usual and the $U_{m n}^{(\tilde{t})}$ are the elements of the orthogonal squark-mixing matrix

$$
U^{(\tilde{t})}=\left(\begin{array}{cc}
U_{11}^{(\tilde{t})} & U_{12}^{(\tilde{t})} \\
U_{21}^{(\tilde{t})} & U_{22}^{(\tilde{t})}
\end{array}\right) .
$$

The relative minus sign between the diagrams $(\beta)$ and $(\gamma)$ of Fig. 14 stems from the interchange of two fermionic operators.

The amplitude in Eq. (22) has now, following the methods explained in Refs. [47, 48], to be projected onto the spin- 0 state using the Clebsch-Gordon coefficients $\langle 0,0 ; s, \bar{s}\rangle$, where the squared brackets of Eq. (22) become due to the antisymmetry of the spin function

$$
\begin{aligned}
& \sum_{s, \bar{s}}\left[u_{\bar{s}}\left(\frac{P}{2}, m_{\tilde{g}}\right) \bar{v}_{s}\left(\frac{P}{2}, m_{\tilde{g}}\right)-u_{s}\left(\frac{P}{2}, m_{\tilde{g}}\right) \bar{v}_{\bar{s}}\left(\frac{P}{2}, m_{\tilde{g}}\right)\right]\langle 0,0 ; s, \bar{s}\rangle \\
= & 2 \sum_{s, \bar{s}}\left[u_{\bar{s}}\left(\frac{P}{2}, m_{\tilde{g}}\right) \bar{v}_{s}\left(\frac{P}{2}, m_{\tilde{g}}\right)\right]\langle 0,0 ; s, \bar{s}\rangle .
\end{aligned}
$$



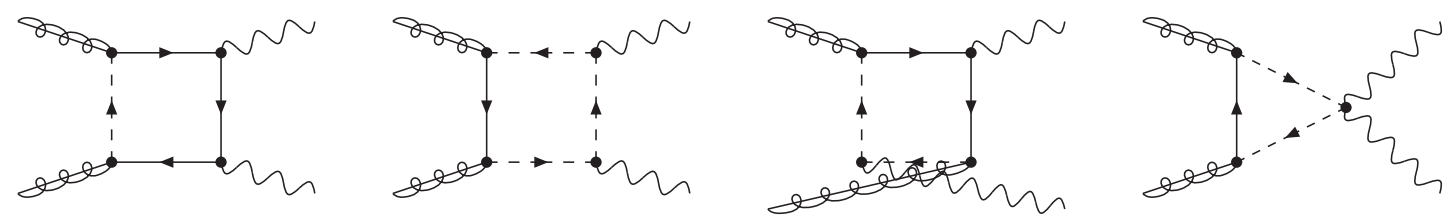

Figure 15: Representative diagrams for the decay into two photons at LO.

Now the calculation can be done as for the case of the decay into two gluons and the rate reads

$$
\Gamma\left(0^{-+} \rightarrow t \bar{t}\right)=\frac{C_{F} \alpha_{s}^{2}|R(0)|^{2}}{2 m_{\tilde{g}}^{2}} \sqrt{1-\frac{m_{t}^{2}}{m_{\tilde{g}}^{2}}}\left(\sum_{h=1}^{2} \frac{m_{\tilde{g}}\left(m_{t}-2 m_{\tilde{g}} U_{h 1}^{(\tilde{t})} U_{h 2}^{(\tilde{t})}\right)}{m_{\tilde{g}}^{2}+m_{\tilde{t}_{h}}^{2}-m_{t}^{2}}\right)^{2} .
$$

\section{B.2 Decay into photons}

Treating all the virtual quarks in Fig. 15 as massless, the onto spin and color-projected amplitude has the form

$$
\begin{aligned}
& \mathcal{A}\left((\tilde{g} \tilde{g})_{1_{S}} \rightarrow \gamma \gamma\right) \\
= & -i T_{F} \sqrt{N_{C}^{2}-1} \frac{e^{2} g^{2}}{\sqrt{2} \pi^{2}} \varepsilon_{\alpha \beta \gamma \delta} k_{1}^{\alpha} k_{2}^{\beta} \epsilon_{1}^{\gamma} \epsilon_{2}^{\delta} \sum_{f, h} Q_{f}^{2} \\
\times \quad & \frac{C_{0}\left(0,-m_{\tilde{g}}^{2}, m_{\tilde{g}}^{2}, m_{q_{f}}^{2}, m_{q_{f}}^{2}, m_{\tilde{q}_{f, h}}^{2}\right)-C_{0}\left(4 m_{\tilde{g}}^{2}, 0,0, m_{q_{f}}^{2}, m_{q_{f}}^{2}, m_{q_{f}}^{2}\right)}{m_{\tilde{g}}^{2}+m_{\tilde{q}_{f, h}}^{2}-m_{q_{f}}^{2}} \\
& \times\left(m_{q_{f}}-2 U_{h 1}^{(\tilde{f})} U_{h 2}^{(\tilde{f})} m_{\tilde{g}}\right) m_{q_{f}} \\
& \left.+\frac{m_{\tilde{q}_{f, h}}^{2} C_{0}\left(0,-m_{\tilde{g}}^{2}, m_{\tilde{g}}^{2}, m_{\tilde{q}_{f, h}}^{2}, m_{\tilde{q}_{f, h}}^{2}, m_{q_{f}}^{2}\right)-m_{q_{f}}^{2} C_{0}\left(0,-m_{\tilde{g}}^{2}, m_{\tilde{g}}^{2}, m_{q_{f}}^{2}, m_{q_{f}}^{2}, m_{\tilde{q}_{f, h}}^{2}\right)}{2\left(m_{\tilde{q}_{f, h}}^{2}-m_{q_{f}}^{2}\right)}\right\} .
\end{aligned}
$$

The sum in the first equation of (26) goes over the flavors $(f)$ and the indices of the squarks $(h)$. The $k_{i}$ are the momenta of the outgoing photons, the $\epsilon_{i}$ their polarization vectors and the $Q_{f}$ the charges of the corresponding quarks and squarks. The definition of the $C_{0}$-functions is the same as in Ref. [49].

\section{References}

[1] W. Y. Keung and A. Khare, Phys. Rev. D 29 (1984) 2657. 
[2] J. H. Kühn and S. Ono, Phys. Lett. B 142 (1984) 436.

[3] J. T. Goldman and H. Haber, Physica 15D, 181 (1985).

[4] V. G. Kartvelishvili, A. V. Tkabladze and E. G. Chikovani, Sov. J. Nucl. Phys. 51, 546 (1990) [Yad. Fiz. 51, 859 (1990)].

[5] E. Chikovani, V. Kartvelishvili, R. Shanidze and G. Shaw, Phys. Rev. D 53, 6653 (1996) arXiv:hep-ph/9602249.

[6] V. G. Kartvelishvili, A. V. Tkabladze and E. G. Chikovani, Z. Phys. C 43 (1989) 509.

[7] N. Arkani-Hamed and S. Dimopoulos, JHEP 0506, 073 (2005) arXiv:hep-th/0405159.

[8] G. F. Giudice and A. Romanino, Nucl. Phys. B 699, 65 (2004) [Erratum-ibid. B 706, 65 (2005)] arXiv:hep-ph/0406088].

[9] W. Kilian, T. Plehn, P. Richardson and E. Schmidt, Eur. Phys. J. C 39, 229 (2005) arXiv:hep-ph/0408088.

[10] K. Cheung and W. Y. Keung, Phys. Rev. D 71, 015015 (2005) arXiv:hep-ph/0408335.

[11] M. Peter, Phys. Rev. Lett. 78, 602 (1997) arXiv:hep-ph/9610209.

[12] M. Peter, Nucl. Phys. B 501, 471 (1997) arXiv:hep-ph/9702245.

[13] Y. Schröder, Phys. Lett. B 447, 321 (1999) arXiv:hep-ph/9812205.

[14] A. V. Smirnov, V. A. Smirnov and M. Steinhauser, Phys. Lett. B 668 (2008) 293 arXiv:0809.1927 [hep-ph]].

[15] S. N. Gupta and S. F. Radford, Phys. Rev. D 24, 2309 (1981).

[16] S. N. Gupta and S. F. Radford, Phys. Rev. D 25, 3430 (1982).

[17] S. Titard and F. J. Yndurain, Phys. Rev. D 49, 6007 (1994) arXiv:hep-ph/9310236.

[18] B. A. Kniehl, A. A. Penin, M. Steinhauser and V. A. Smirnov, Phys. Rev. D 65, 091503 (2002) arXiv:hep-ph/0106135.

[19] A. Pineda and F. J. Yndurain, Phys. Rev. D 58, 094022 (1998) arXiv:hep-ph/9711287.

[20] A. Pineda and J. Soto, Nucl. Phys. Proc. Suppl. 64, 428 (1998) arXiv:hep-ph/9707481. 
[21] A. V. Manohar and I. W. Stewart, Phys. Rev. D 62, 074015 (2000) arXiv:hep-ph/0003032.

[22] M. Beneke, Phys. Lett. B 434 (1998) 115 arXiv:hep-ph/9804241.

[23] A. H. Hoang, M. C. Smith, T. Stelzer and S. Willenbrock, Phys. Rev. D 59 (1999) 114014 arXiv:hep-ph/9804227.

[24] J. H. Kühn, A. A. Penin and A. A. Pivovarov, Nucl. Phys. B 534, 356 (1998) arXiv:hep-ph/9801356.

[25] A. A. Penin and M. Steinhauser, Phys. Lett. B 538 (2002) 335 arXiv:hep-ph/0204290.

[26] B. A. Kniehl, A. A. Penin, V. A. Smirnov and M. Steinhauser, Nucl. Phys. B 635, 357 (2002) arXiv:hep-ph/0203166.

[27] V. S. Fadin and V. A. Khoze, JETP Lett. 46, 525 (1987) [Pisma Zh. Eksp. Teor. Fiz. 46, 417 (1987)].

[28] J. H. Kühn and P. M. Zerwas, Phys. Rept. 167, 321 (1988).

[29] M. J. Strassler and M. E. Peskin, Phys. Rev. D 43, 1500 (1991).

[30] Y. Sumino, K. Fujii, K. Hagiwara, H. Murayama and C. K. Ng, Phys. Rev. D 47, 56 (1993).

[31] M. Jezabek, J. H. Kühn and T. Teubner, Z. Phys. C 56, 653 (1992).

[32] A. H. Hoang et al., Eur. Phys. J. direct C 2, 1 (2000) arXiv:hep-ph/0001286.

[33] A. H. Hoang and C. J. Reisser, Phys. Rev. D $71 \quad$ (2005) 074022 arXiv:hep-ph/0412258.

[34] V. S. Fadin, V. A. Khoze and T. Sjöstrand, Z. Phys. C 48, 613 (1990).

[35] K. Hagiwara, Y. Sumino and H. Yokoya, Phys. Lett. B 666, 71 (2008) arXiv:0804.1014 [hep-ph]].

[36] Y. Kiyo, J. H. Kuhn, S. Moch, M. Steinhauser and P. Uwer, Eur. Phys. J. C 60 (2009) 375 [arXiv:0812.0919 [hep-ph]].

[37] M. Melles, Phys. Rev. D 58, 114004 (1998) arXiv:hep-ph/9805216.

[38] M. Melles, Phys. Rev. D 62, 074019 (2000) arXiv:hep-ph/0001295.

[39] M. Beneke, Y. Kiyo and K. Schuller, Nucl. Phys. B 714, 67 (2005) arXiv:hep-ph/0501289. 
[40] A. A. Penin, V. A. Smirnov and M. Steinhauser, Nucl. Phys. B 716 (2005) 303 arXiv:hep-ph/0501042.

[41] Y. Kiyo and Y. Sumino, Phys. Rev. D 67 (2003) 071501 arXiv:hep-ph/0211299].

[42] H. E. Haber and G. L. Kane, Phys. Rept. 117, 75 (1985).

[43] J. H. Kühn and E. Mirkes, Phys. Rev. D 48, 179 (1993) arXiv:hep-ph/9301204].

[44] A. D. Martin, W. J. Stirling, R. S. Thorne and G. Watt, arXiv:0901.0002 [hep-ph].

[45] The CMS Collaboration, CMS PAS SBM 07001.

[46] K. Hagiwara and H. Yokoya, arXiv:0909.3204 [hep-ph].

[47] B. Guberina, J. H. Kühn, R. D. Peccei and R. Rückl, Nucl. Phys. B 174, 317 (1980).

[48] J. H. Kühn, J. Kaplan and E. G. O. Safiani, Nucl. Phys. B 157, 125 (1979).

[49] G. 't Hooft and M. J. G. Veltman, Nucl. Phys. B 153, 365 (1979). 